TRANSACTIONS OF THE

AMERICAN MATHEMATICAL SOCIETY

Volume 363, Number 4, April 2011, Pages 1855-1886

S 0002-9947(2010)05137-2

Article electronically published on November 16, 2010

\title{
MULTIPLIERS AND INTEGRATION OPERATORS ON DIRICHLET SPACES
}

\author{
PETROS GALANOPOULOS, DANIEL GIRELA, AND JOSÉ ÁNGEL PELÁEZ
}

\begin{abstract}
For $0<p<\infty$ and $\alpha>-1$, we let $\mathcal{D}_{\alpha}^{p}$ denote the space of those functions $f$ which are analytic in the unit disc $\mathbb{D}$ in $\mathbb{C}$ and satisfy $\int_{\mathbb{D}}\left(1-|z|^{2}\right)^{\alpha}\left|f^{\prime}(z)\right|^{p} d x d y<\infty$. Of special interest are the spaces $\mathcal{D}_{p-1}^{p}$ $(0<p<\infty)$ which are closely related with Hardy spaces and the analytic Besov spaces $B^{p}=\mathcal{D}_{p-2}^{p}(1<p<\infty)$. A good number of results on the boundedness of integration operators and multipliers from $\mathcal{D}_{\alpha}^{p}$ to $\mathcal{D}_{\beta}^{q}$ are known in the case $p<q$. Here we are mainly concerned with the upper triangle case $0<q \leq p$. We describe the boundedness of these operators from $\mathcal{D}_{\alpha}^{p}$ to $\mathcal{D}_{\beta}^{q}$ in the case $0<q<p$. Among other results we prove that if $0<q<p$ and $\frac{p \beta-q \alpha}{p-q} \leq-1$, then the only pointwise multiplier from $\mathcal{D}_{\alpha}^{p}$ to $\mathcal{D}_{\beta}^{q}$ is the trivial one. In particular, we have that 0 is the only multiplier from $\mathcal{D}_{p-1}^{p}$ to $\mathcal{D}_{q-1}^{q}$ if $p \neq q$, and from $B^{p}$ to $B^{q}$ if $1<q<p$. Also, we give a number of explicit examples of multipliers from $\mathcal{D}_{\alpha}^{p}$ to $\mathcal{D}_{\beta}^{q}$ in the remaining case $\frac{p \beta-q \alpha}{p-q}>-1$. Furthermore, we present a number of results on the self-multipliers of $\mathcal{D}_{\alpha}^{p}$ $(0<p<\infty, \alpha>-1)$. We prove that 0 is the only compact multiplier from $\mathcal{D}_{p-1}^{p}$ to itself $(0<p<\infty)$ and we give a number of explicit examples of functions which are self-multipliers of $\mathcal{D}_{\alpha}^{p}$.

We also consider the closely related question of characterizing the Carleson measures for the spaces $\mathcal{D}_{\alpha}^{p}$. In particular, we prove constructively that a result of Arcozzi, Rochberg and Sawyer characterizing the Carleson measures for $\mathcal{D}_{\alpha}^{p}$ in the range $-1<\alpha<p-1$ cannot be extended to cover the case $\alpha=p-1$ and we find a certain condition on a measure $\mu$ which is necessary for $\mu$ to be a $q$-Carleson measure for $\mathcal{D}_{\alpha}^{p}(0<q<p, \alpha>-1)$. This result plays a basic role in our work concerning integration operators.
\end{abstract}

\section{INTRODUCTION}

If $f$ and $g$ are analytic functions in the unit disc $\mathbb{D}$, we set

$$
I_{g}(f)(z)=\int_{0}^{z} g(\xi) f^{\prime}(\xi) d \xi, J_{g}(f)(z)=\int_{0}^{z} g^{\prime}(\xi) f(\xi) d \xi, z \in \mathbb{D} \text {. }
$$

Received by the editors September 23, 2008.

2010 Mathematics Subject Classification. Primary 30H20, 30H99, 47B38.

Key words and phrases. Carleson measures, multiplication operators, integration operators, Dirichlet spaces, Bergman spaces.

This research was partially supported by grants from "the Ministerio de Educación y Ciencia, Spain" (MTM2007-60854, MTM2007-30904-E and Ingenio Mathematica (i-MATH) No. CSD2006-00032); from "La Junta de Andalucía" (FQM210 and P06-FQM01504) and from the European Networking Programme "HCAA" of the European Science Foundation. 
We also set $M_{g}(f)=g f$. In this paper we are interested in studying the integration operators $I_{g}, J_{g}$ and the multiplication operators $M_{g}$ acting on the spaces of analytic functions in $\mathbb{D}$ of Dirichlet type $\mathcal{D}_{\alpha}^{p}(0<p<\infty, \alpha>-1)$ and, also, in the closely related question of characterizing the Carleson measures for these spaces. Of special interest are the spaces $\mathcal{D}_{p-1}^{p}(0<p<\infty)$ which are closely related with Hardy spaces and the conformally-invariant analytic Besov spaces $B^{p}=\mathcal{D}_{p-2}^{p}$ $(1<p<\infty)$.

A good number of results about the boundedness of the mentioned operators from $\mathcal{D}_{\alpha}^{p}$ to $\mathcal{D}_{\beta}^{q}$ are known in the case $p<q$. In this paper we shall be mainly concerned with the upper triangle case $0<q \leq p$. We obtain a complete characterization of the boundedness of $I_{g}, J_{g}$ and $M_{g}$ from $\mathcal{D}_{\alpha}^{p}$ to $\mathcal{D}_{\beta}^{q}$ in the case $0<q<p, \alpha, \beta>-1$. Among other results we shall prove that for a wide range of the parameters $p, \alpha, q, \beta$ the only multiplier from $\mathcal{D}_{\alpha}^{p}$ to $\mathcal{D}_{\beta}^{q}$ is the trivial one. In particular, we have:

(i) If $0<p, q<\infty$ and $p \neq q$, then the only multiplier from $\mathcal{D}_{p-1}^{p}$ to $\mathcal{D}_{q-1}^{q}$ is identically zero. Furthermore, the only compact multiplier from $\mathcal{D}_{p-1}^{p}$ to itself $(0<p<\infty)$ is also the trivial one.

(ii) If $1<q<p$, then 0 is the only multiplier mapping $B^{p}$ to $B^{q}$.

We also present a number of explicit examples of nontrivial multipliers for the other values of the parameters.

Carleson measures arise in many questions involving analysis in function spaces. In particular, they play a basic role in studying the boundedness of the operators mentioned above. Characterizations of the $q$-Carleson measures for the space $\mathcal{D}_{\alpha}^{p}$ are known for certain values of the parameters $p, q, \alpha$ but some cases remain open. Specifically, we mention that it is an open question to find a characterization of the $q$-Carleson measures for the space $\mathcal{D}_{p-1}^{p}$ in the cases $q<p$ and $p=q>2$. In this paper we obtain a number of results which give some light on these questions and which are also useful in our study of the boundedness of operators between distinct spaces of Dirichlet type. In particular, we prove constructively that a result of Arcozzi, Rochberg and Sawyer characterizing the Carleson measures for $\mathcal{D}_{\alpha}^{p}$ in the range $p>1,-1<\alpha<p-1$ cannot be extended to cover the case $\alpha=p-1$. We also find a certain condition on a measure $\mu$ which is necessary for $\mu$ to be a $q$-Carleson measure for $\mathcal{D}_{\alpha}^{p}(0<q<p, \alpha>-1)$. This result plays a basic role in our work concerning the integration operators. In order to prove the necessity of our condition we use a decomposition theorem for the spaces $\mathcal{D}_{\alpha}^{p}$ which is based on one of Rochberg for Bergman spaces and may be of independent interest.

We close this section by noticing that, as usual, we shall be using the convention that $C_{p, \alpha, q, \beta} \ldots$ will denote a positive constant which depends only upon the displayed parameters $p, \alpha, q, \beta \ldots$ (which sometimes will be omitted) but not necessarily the same at different occurrences. Also, throughout the paper, if $p, q>1$, then $p^{\prime}$ and $q^{\prime}$ denote the "exponents conjugate" to $p$ and $q$, respectively, that is, $\frac{1}{p}+\frac{1}{p^{\prime}}=\frac{1}{q}+\frac{1}{q^{\prime}}=1$.

\section{Statement of the main Results}

1.1. Multipliers and integration operators between Dirichlet spaces. Let $\mathbb{D}=\{z \in \mathbb{C}:|z|<1\}$ denote the open unit disc of the complex plane $\mathbb{C}$ and let $\mathcal{H o l}(\mathbb{D})$ be the space of all analytic functions in $\mathbb{D}$. 
If $0<r<1$ and $f \in \mathcal{H}$ ol $(\mathbb{D})$, we set

$$
\begin{gathered}
M_{p}(r, f)=\left(\frac{1}{2 \pi} \int_{0}^{2 \pi}\left|f\left(r e^{i t}\right)\right|^{p} d t\right)^{1 / p}, 0<p<\infty, \\
M_{\infty}(r, f)=\sup _{|z|=r}|f(z)| .
\end{gathered}
$$

If $0<p \leq \infty$, then the Hardy space $H^{p}$ consists of those $f \in \mathcal{H o l}(\mathbb{D})$ such that $\|f\|_{H^{p}} \stackrel{\text { def }}{=} \sup _{0<r<1} M_{p}(r, f)<\infty$ (see [12] for the theory of $H^{p}$-spaces). If $0<p<\infty$ and $\alpha>-1$, the weighted Bergman space $A_{\alpha}^{p}$ consists of those $f \in \mathcal{H o l}(\mathbb{D})$ such that

$$
\|f\|_{A_{\alpha}^{p}} \stackrel{\text { def }}{=}\left((\alpha+1) \int_{\mathbb{D}}(1-|z|)^{\alpha}|f(z)|^{p} d A(z)\right)^{1 / p}<\infty .
$$

The unweighted Bergman space $A_{0}^{p}$ is simply denoted by $A^{p}$. Here, $d A(z)=\frac{1}{\pi} d x d y$ denotes the normalized Lebesgue area measure in $\mathbb{D}$. We refer to [13, 22] and [36] for the theory of these spaces.

The space $\mathcal{D}_{\alpha}^{p}(0<p<\infty, \alpha>-1)$ consists of those $f \in \mathcal{H}$ ol $(\mathbb{D})$ such that $f^{\prime} \in A_{\alpha}^{p}$. Hence, if $f$ is analytic in $\mathbb{D}$, then $f \in \mathcal{D}_{\alpha}^{p}$ if and only if

$$
\|f\|_{\mathcal{D}_{\alpha}^{p}}^{p} \stackrel{\text { def }}{=}|f(0)|^{p}+\left\|f^{\prime}\right\|_{A_{\alpha}^{p}}^{p}<\infty .
$$

If $p<\alpha+1$, then it is well known that $\mathcal{D}_{\alpha}^{p}=A_{\alpha-p}^{p}$ (see, e.g., Theorem 6 of [15]). On the other hand, if $p>\alpha+2$, then $\mathcal{D}_{\alpha}^{p} \subset H^{\infty}$. Therefore the interesting ranges for $(p, \alpha)$ are $\alpha+1 \leq p \leq \alpha+2$. It is trivial that $D_{1}^{2}=H^{2}$ and we have $H^{p} \subset \mathcal{D}_{p-1}^{p}$ for $2 \leq p<\infty$ [23], and $\mathcal{D}_{p-1}^{p} \subset H^{p}$ for $0<p \leq 2$ [15, 31]. For $p>1$, the space $\mathcal{D}_{p-2}^{p}$ is the analytic Besov space $B^{p}$. The spaces $B^{p}, 1<p<\infty$, form a nested scale of conformally-invariant spaces which are contained in $V M O A$ and show up naturally in different settings (see [4], [10] and [37]). In particular, $\mathcal{D}_{0}^{2}=B^{2}$ is the classical Dirichlet space.

In this paper we shall focus our attention to studying the boundedness and compactness of a number of important operators acting between distinct Dirichlet spaces.

For $g$ analytic in $\mathbb{D}$, the integration operators $I_{g}$ and $J_{g}$ are defined as follows:

$$
\begin{aligned}
& I_{g}(f)(z) \stackrel{\text { def }}{=} \int_{0}^{z} g(\xi) f^{\prime}(\xi) d \xi, \quad f \in \mathcal{H} o l(\mathbb{D}), \quad z \in \mathbb{D}, \\
& J_{g}(f)(z) \stackrel{\text { def }}{=} \int_{0}^{z} g^{\prime}(\xi) f(\xi) d \xi, \quad f \in \mathcal{H} \text { ol }(\mathbb{D}), \quad z \in \mathbb{D} .
\end{aligned}
$$

The integration operators $I_{g}$ and $J_{g}$ have been studied in a good number of papers. Let us just mention here that Aleman and Cima characterized in [2] those $g \in \mathcal{H o l}(\mathbb{D})$ for which $J_{g}$ maps $H^{p}$ into $H^{q}$, and that Aleman and Siskakis studied in 3] the operators $J_{g}$ acting on Bergman spaces. A lot of information about the operators $J_{g}$ acting on Hardy and Bergman spaces and in other related spaces can be found in the recent survey paper [1].

For $g \in \mathcal{H} o l(\mathbb{D})$, the multiplication operator $M_{g}$ is defined by

$$
M_{g}(f)(z) \stackrel{\text { def }}{=} g(z) f(z), \quad f \in \mathcal{H o l}(\mathbb{D}), z \in \mathbb{D} .
$$

If $X$ and $Y$ are two spaces of analytic functions in $\mathbb{D}$ (which will always be assumed to be Banach or $F$-spaces continuously embedded in $\mathcal{H} o l(\mathbb{D}))$ and if $g \in$ 
$\mathcal{H} o l(\mathbb{D})$, then $g$ is said to be a multiplier from $X$ to $Y$ if $M_{g}(X) \subset Y$. The space of all multipliers from $X$ to $Y$ will be denoted by $M(X, Y)$ and $M(X)$ will stand for $M(X, X)$. Using the closed graph theorem we see that for any of the three operators $I_{g}, J_{g}, M_{g}$, we have that if it applies $X$ into $Y$, then it is continuous from $X$ to $Y$. We remark also that

$$
I_{g}(f)+J_{g}(f)=M_{g}(f)-f(0) g(0) .
$$

Thus if two of the operators $I_{g}, J_{g}, M_{g}$ are bounded from $X$ to $Y$ so is the third one.

Girela and Peláez [19] studied the boundedness of these operators from $\mathcal{D}_{\alpha}^{p}$ to $\mathcal{D}_{\beta}^{q}$ in the lower triangle case $(0<p<q)$ and, among others, they obtained the following results.

Theorem A. Suppose that $g \in \mathcal{H} o l(\mathbb{D}), 0<p<q$ and $\alpha, \beta>-1$. If $p>\alpha$, then the following conditions are equivalent:

(i) $I_{g}$ is a bounded operator from $\mathcal{D}_{\alpha}^{p}$ to $\mathcal{D}_{\beta}^{q}$;

(ii) $M_{\infty}(r, g)=\mathrm{O}\left((1-r)^{\frac{2+\alpha}{p}-\frac{\beta+2}{q}}\right)$.

Theorem B. Suppose that $g \in \mathcal{H o l}(\mathbb{D}), 0<p<q$ and $\alpha, \beta>-1$. If $p-2<\alpha<p$, and $\frac{2+\alpha}{p}-\frac{\beta+2}{q}>0$, the following conditions are equivalent:

(i) $I_{g}: \mathcal{D}_{\alpha}^{p} \rightarrow \mathcal{D}_{\beta}^{q}$ is a bounded operator;

(ii) $M_{g}: \mathcal{D}_{\alpha}^{p} \rightarrow \mathcal{D}_{\beta}^{q}$ is a bounded operator;

(iii) $g \equiv 0$.

In particular, if $0<p<q$, then $M\left(\mathcal{D}_{p-1}^{p}, \mathcal{D}_{q-1}^{q}\right)=\{0\}$.

In this paper we are interested in the upper triangle case, $0<q \leq p$. Let us start with the case $0<q<p$. We shall prove the following results.

Theorem 1. Suppose that $g \in \mathcal{H} o l(\mathbb{D}), 0<q<p, \alpha, \beta>-1$ and $\frac{p \beta-q \alpha}{p-q}>-1$. Then:

(1) $I_{g}$ maps $\mathcal{D}_{\alpha}^{p}$ to $\mathcal{D}_{\beta}^{q}$ if and only if $g \in A_{\frac{p \beta-q \alpha}{p-q}}^{\frac{p q}{p-q}}$.

(2) If $g \in M\left(\mathcal{D}_{\alpha}^{p}, \mathcal{D}_{\beta}^{q}\right)$, then $g \in A_{\frac{p \beta-q \alpha}{p-q}}^{\frac{p q}{p-q}}$.

(3) If $J_{g}$ maps $\mathcal{D}_{\alpha}^{p}$ to $\mathcal{D}_{\beta}^{q}$, then $g \in A_{\frac{p \beta-q \alpha}{p-q}}^{\frac{p q}{p-q}}$.

(4) The following two conditions are equivalent:

(a) $M_{g}$ maps $\mathcal{D}_{\alpha}^{p}$ to $\mathcal{D}_{\beta}^{q}$;

(b) $J_{g} \operatorname{maps} \mathcal{D}_{\alpha}^{p}$ to $\mathcal{D}_{\beta}^{q}$.

Theorem 2. Suppose that $g \in \mathcal{H} o l(\mathbb{D}), 0<q<p, \alpha, \beta>-1$ and $\frac{p \beta-q \alpha}{p-q} \leq-1$. Then the following conditions are equivalent:

(1) $I_{g}$ maps $\mathcal{D}_{\alpha}^{p}$ to $\mathcal{D}_{\beta}^{q}$;

(2) $M_{g}$ maps $\mathcal{D}_{\alpha}^{p}$ to $\mathcal{D}_{\beta}^{q}$;

(3) $g \equiv 0$.

We remark that part (4) of Theorem 1 does not remain true for $\frac{p \beta-q \alpha}{p-q} \leq-1$. Indeed, it is trivial that if $g$ is constant and not identically zero, then $J_{g} \equiv 0$ and, hence, $J_{g}\left(\mathcal{D}_{\alpha}^{p}\right) \subset \mathcal{D}_{\beta}^{q}$; however, Theorem 2 implies that $g \notin M\left(\mathcal{D}_{\alpha}^{p}, \mathcal{D}_{\beta}^{q}\right)$ in this case. Nonconstant examples also can be given. For example, Theorem 2 implies that 
$M\left(H^{2}, \mathcal{D}_{0}^{1}\right)=\{0\}$ but it is easy to see that if $0<\alpha \leq 1$ and $g$ belongs to the mean-Lipschitz space $\Lambda_{\alpha}^{2}$ (see [12, Chapter 5]), then $J_{g}\left(H^{2}\right) \subset \mathcal{D}_{0}^{1}$.

We remark also that, using the convention that $A_{\gamma}^{p}=\{0\}$ if $\gamma \leq-1$, Theorem 2 can be thought of as saying that conclusions (1) and (2) of Theorem 1 hold whenever $0<q<p$ and $\alpha, \beta>-1$.

Next we shall give explicit examples of nontrivial multipliers from $\mathcal{D}_{\alpha}^{p}$ into $\mathcal{D}_{\beta}^{q}$ in the case $0<q<p$ and $\frac{p \beta-q \alpha}{p-q}>-1$. First of all, let us remark that if $\alpha>p-1$, then we also have $\beta>q-1$ and then $\mathcal{D}_{\alpha}^{p}=A_{\alpha-p}^{p}, \mathcal{D}_{\beta}^{q}=A_{\beta-q}^{q}$. Then, Theorem 1 (iv) of [35] yields

$$
M\left(\mathcal{D}_{\alpha}^{p}, \mathcal{D}_{\beta}^{q}\right)=M\left(A_{\alpha-p}^{p}, A_{\beta-q}^{q}\right)=A_{\frac{p \beta-q \alpha}{p-q}}^{\frac{p q}{p-q}}, \quad 0<q<p, \alpha>p-1, \beta>q-1 .
$$

Turning to the case $p-2 \leq \alpha \leq p-1$, we shall study the question of characterizing the power series with Hadamard gaps which belong to $M\left(\mathcal{D}_{\alpha}^{p}, \mathcal{D}_{\beta}^{q}\right)$ in this range of the parameters. Before stating our results it is worth recalling the characterization of the power series with Hadamard gaps in the spaces $\mathcal{D}_{\alpha}^{p}$, a result which can be deduced from Proposition 2.1 of [7.

Proposition A. Suppose that $0<p<\infty, \alpha>-1$ and that $f$ is an analytic function in $\mathbb{D}$ which is given by a power series with Hadamard gaps,

$$
f(z)=\sum_{k=1}^{\infty} a_{k} z^{n_{k}} \quad(z \in \mathbb{D}) \text { with } n_{k+1} \geq \lambda n_{k} \text { for all } k \quad(\lambda>1) .
$$

Then,

$$
f \in \mathcal{D}_{\alpha}^{p} \Longleftrightarrow \sum_{k=1}^{\infty} n_{k}^{p-\alpha-1}\left|a_{k}\right|^{p}<\infty .
$$

Theorem 3. Suppose that $0<q<p<\infty, \alpha, \beta>-1, \frac{p \beta-q \alpha}{p-q}>-1$ and let $g$ be an analytic function in $\mathbb{D}$ which is given by a power series with Hadamard gaps. If either $p-2 \leq \alpha<p-1$ or $\alpha=p-1$ and $p \leq 2$, then $g \in M\left(\mathcal{D}_{\alpha}^{p}, \mathcal{D}_{\beta}^{q}\right)$ if and only if $g \in \mathcal{D}_{\beta}^{q}$.

Our next result asserts that Theorem 3 cannot be extended to cover the case $\alpha=p-1$ and $p>2$.

Theorem 4. Suppose that $2<p<\infty, 0<q<p$ and $\beta>q-1$. Then there exists $g \in \mathcal{D}_{\beta}^{q}$ which is given by a power series with Hadamard gaps and such that $g \notin M\left(\mathcal{D}_{p-1}^{p}, \mathcal{D}_{\beta}^{q}\right)$.

Joining Theorem $\mathrm{B}$ and Theorem 2 we see that $M\left(\mathcal{D}_{\alpha}^{p}, \mathcal{D}_{\beta}^{q}\right)=\{0\}$ for a wide range of values of the parameters $p, \alpha, q, \beta$. Indeed, we can state the following result.

Corollary 1. (i) Suppose that $0<p, q<\infty, p \neq q, \alpha, \beta>-1, p-1 \leq \alpha$ and $\beta \leq q-1$. Then $M\left(\mathcal{D}_{\alpha}^{p}, \mathcal{D}_{\beta}^{p}\right)=\{0\}$.

In particular: If $0<p<\infty$ and $p \neq q$, then $M\left(\mathcal{D}_{p-1}^{p}, \mathcal{D}_{q-1}^{q}\right)=\{0\}$.

(ii) If $1<q<p<\infty$, then $M\left(B^{p}, B^{q}\right)=\{0\}$.

We note here a difference between Besov spaces and the spaces $\mathcal{D}_{p-1}^{p}$ : If $p<$ $q$, then $B^{p} \subset B^{q}$ and, hence, $M\left(B^{p}, B^{q}\right) \neq\{0\}$; however, if $p \neq q$ there is no relation of inclusion between $\mathcal{D}_{p-1}^{p}$ and $\mathcal{D}_{q-1}^{q}$ and, in fact, we have proved that $M\left(\mathcal{D}_{p-1}^{p}, \mathcal{D}_{q-1}^{q}\right)=\{0\}$ in this case. 
We shall also prove the following extension of Corollary 1 concerning the case $p=q$.

Theorem 5. Suppose $0<p<\infty, \alpha, \beta>-1$. If either $p-1 \leq \alpha$ and $\beta<p-1$ or $p-1<\alpha$ and $\beta \leq p-1$, then $M\left(\mathcal{D}_{\alpha}^{p}, \mathcal{D}_{\beta}^{p}\right)=\{0\}$.

Certainly, for any $p \in(0, \infty)$ we have that $M\left(\mathcal{D}_{p-1}^{p}, \mathcal{D}_{p-1}^{p}\right)=M\left(\mathcal{D}_{p-1}^{p}\right) \neq\{0\}$; it is trivial that any constant function belongs to $M\left(\mathcal{D}_{p-1}^{p}\right)$. Wu gave in [34, Theorem 4.2] a characterization $M\left(\mathcal{D}_{p-1}^{p}\right)$ in terms of Carleson measures (see Section 1.2 below), and a number of explicit nontrivial elements of $M\left(\mathcal{D}_{p-1}^{p}\right)$ are known. For instance:

- Vinogradov proved in 31] that the atomic singular inner function

$$
S_{\gamma, \eta}(z)=\exp \left(\gamma \frac{z+\eta}{z-\eta}\right), \quad z \in \mathbb{D}
$$

where $\gamma>0$ and $\eta \in \partial \mathbb{D}$, belongs to $M\left(\mathcal{D}_{p-1}^{p}\right)$, whenever $0<p \leq 2$. He also gave a number of conditions on the zeros of a Blaschke product $B$ under which $B \in M\left(\mathcal{D}_{p-1}^{p}\right)(0<p<2)$.

- Girela and Peláez gave in Section 5 of 18 several other examples of multipliers of $\mathcal{D}_{p-1}^{p}, 0<p<2$. In particular, Theorem 5.2 of [18] asserts that if $0<q \leq 1$, $q \leq p<2$ and $g$ is an analytic function in $\mathbb{D}$ which is given by a power series with Hadamard gaps, $g(z)=\sum_{k=1}^{\infty} a_{k} z^{n_{k}}(z \in \mathbb{D})$, with $\sum_{k=1}^{\infty}\left|a_{k}\right|^{q}<\infty$, then $g \in M\left(\mathcal{D}_{p-1}^{p}\right)$. The following theorem contains an improvement of this result and is also an extension of Theorem 3 .

Theorem 6. Suppose that $0<p<\infty, \alpha>-1$ and let $g$ be an analytic function in $\mathbb{D}$ which is given by a power series with Hadamard gaps. We have:

(i) If $p-1<\alpha$, then $g \in M\left(\mathcal{D}_{\alpha}^{p}\right)$ if and only if $g \in H^{\infty}$.

(ii) If $\alpha<p-1$, then $g \in M\left(\mathcal{D}_{\alpha}^{p}\right)$ if and only if $g \in \mathcal{D}_{\alpha}^{p}$.

(iii) If $1<p \leq 2$ and $\alpha=p-1$, then $g \in M\left(\mathcal{D}_{\alpha}^{p}\right)$ if and only if $g \in H^{\infty}$.

(iv) If $0<p \leq 1$ and $\alpha=p-1$, then $g \in M\left(\mathcal{D}_{\alpha}^{p}\right)$ if and only if $g \in \mathcal{D}_{\alpha}^{p}$.

We recall that if $g \in M\left(\mathcal{D}_{\alpha}^{p}\right)$, then $g \in H^{\infty} \cap \mathcal{D}_{\alpha}^{p}$ (see, e.g., [31, Lemma 1.10] and use the fact that $\mathcal{D}_{\alpha}^{p}$ contains the constant functions). Theorem 6 shows that, except in the case $\alpha=p-1$ and $p>2$, for a function $g$ given by a power series with Hadamard gaps the condition $g \in H^{\infty} \cap \mathcal{D}_{\alpha}^{p}$ is also sufficient to assert that $g \in M\left(\mathcal{D}_{\alpha}^{p}\right)$.

It is natural to ask whether or not part (iii) of Theorem [6 remains true for $p>2$. We shall show that the answer is negative (note that if $p>2$, then $H^{\infty} \cap \mathcal{D}_{p-1}^{p}=H^{\infty}$ since $H^{p} \subset \mathcal{D}_{p-1}^{p}$ for $p>2$ ).

Theorem 7. Suppose that $2<p<\infty$. Then there exists a function $g \in \mathcal{H}$ ol $(\mathbb{D})$ given by a power series with Hadamard gaps such that $g \in H^{\infty} \backslash M\left(\mathcal{D}_{p-1}^{p}\right)$.

Once we know a number of nontrivial examples of multipliers of the space $\mathcal{D}_{p-1}^{p}$, it is natural to ask whether or not there is some nontrivial $g \in \mathcal{H}$ ol $(\mathbb{D})$ such that $M_{g}$ is a compact operator from $\mathcal{D}_{p-1}^{p}$ to itself. Our next result asserts that the answer to this question is negative.

Theorem 8. Suppose that $0<p<\infty$ and $g \in \mathcal{H}$ ol $(\mathbb{D})$. Then $M_{g}$ is a compact operator from $\mathcal{D}_{p-1}^{p}$ to itself if and only if $g \equiv 0$. 
1.2. Carleson measures for Dirichlet spaces. If $I \subset \partial \mathbb{D}$ is an interval, $|I|$ will denote the length of $I$. The Carleson square $S(I)$ is defined as $S(I)=\left\{r e^{i t}: e^{i t} \in\right.$ $\left.I, \quad 1-\frac{|I|}{2 \pi} \leq r<1\right\}$. Also, for $a \in \mathbb{D}$, the Carleson box $S(a)$ is defined by

$$
S(a)=\left\{z \in \mathbb{D}: 1-|z| \leq 1-|a|,\left|\frac{\arg (a \bar{z})}{2 \pi}\right| \leq \frac{1-|a|}{2}\right\} .
$$

We shall also use the modified box

$$
\tilde{S}(a)=\left\{z \in \mathbb{D}: 1-|z| \leq 2(1-|a|),\left|\frac{\arg (a \bar{z})}{2 \pi}\right| \leq \frac{1-|a|}{2}\right\} .
$$

If $s>0$ and $\mu$ is a positive Borel measure on $\mathbb{D}$, we shall say that $\mu$ is an $s$-Carleson measure if there exists a positive constant $C$ such that

$$
\mu(S(I)) \leq C|I|^{s}, \quad \text { for any interval } I \subset \partial \mathbb{D},
$$

or, equivalently, if there exists $C>0$ such that

$$
\mu(S(a)) \leq C(1-|a|)^{s}, \quad \text { for all } a \in \mathbb{D} .
$$

A 1-Carleson measure will be simply called a (classical) Carleson measure.

If $X$ is a subspace of $\mathcal{H o l}(\mathbb{D}), 0<q<\infty$ and $\mu$ is a positive Borel measure in $\mathbb{D}, \mu$ is said to be a " $q$-Carleson measure for the space $X$ " or an " $(X, q)$-Carleson measure" if $X \subset L^{q}(d \mu)$. The $q$-Carleson measures for the spaces $H^{p}, 0<p, q<\infty$ are completely characterized. Carleson proved in his fundamental paper 8 that the $p$-Carleson measures for $H^{p}(0<p<\infty)$ are the (classical) Carleson measures. Duren [11] (see also [12, Theorem 9.4]) extended this result by showing that, for $0<p<q$, the $q$-Carleson measures for $H^{p}$ are precisely the $q / p$-Carleson measures. Luecking [26] solved the remaining case $0<q<p$.

The $q$-Carleson measures for the Bergman space $A_{\alpha}^{p}$ have also been characterized by Luecking [24] in the case $p \leq q$, and in [25] and [27] in the case $q<p$ (see [35, Theorem D]).

Carleson measures arise in many questions involving analytic function spaces. In particular, they play a very important role in studying the boundedness and compactness of operators acting between them:

If $0<p<\infty, \alpha>-1$ and $g \in \mathcal{H o l}(\mathbb{D})$, we let $\mu_{g, p, \alpha}$ be the Borel measure on $\mathbb{D}$ defined by

$$
d \mu_{g, p, \alpha}(z)=\left(1-|z|^{2}\right)^{\alpha}\left|g^{\prime}(z)\right|^{p} d A(z) .
$$

It follows readily from the definitions that:

$J_{g}$ is a bounded operator from $\mathcal{D}_{\alpha}^{p}$ to $\mathcal{D}_{\beta}^{q}$ if and only if the measure $\mu_{g, q, \beta}$ is a $\left(\mathcal{D}_{\alpha}^{p}, q\right)$-Carleson measure.

We can also state the following result.

Theorem $\mathbf{C}$. Let $g$ be an analytic function in $\mathbb{D}$.

(i) If $\alpha>-1$ and $0<p<\infty$, then $g \in M\left(\mathcal{D}_{\alpha}^{p}\right)$ if and only if $g \in H^{\infty}$ and the measure $\mu_{g, p, \alpha}$ is a p-Carleson measure for $\mathcal{D}_{\alpha}^{p}$. if

(ii) If $0<p<q, \alpha, \beta>-1$ and $p-2<\alpha<p$, then $g \in M\left(\mathcal{D}_{\alpha}^{p}, \mathcal{D}_{\beta}^{q}\right)$ if and only

$$
M_{\infty}(r, g)=\mathrm{O}\left((1-r)^{\frac{2+\alpha}{p}-\frac{2+\beta}{q}}\right)
$$

and $\mu_{g, q, \beta}$ is a $\frac{q}{p}(\alpha-p+2)$-Carleson measure.

(iii) If $0<q<p, \alpha, \beta>-1$ and $\frac{p \beta-q \alpha}{p-q}>-1$, then $g \in M\left(\mathcal{D}_{\alpha}^{p}, \mathcal{D}_{\beta}^{q}\right)$ if and only if the measure $\mu_{g, q, \beta}$ is a $\left(\mathcal{D}_{\alpha}^{p}, q\right)$-Carleson measure. 
Here: (i) comes from [31, Lemma 1.10], (see also [34, Theorem 4.2] and [5, Theorem 15]); (ii) is Theorem 3 of [19] and (iii) follows from Theorem 1 .

We shall obtain a number of results about the $\left(\mathcal{D}_{\alpha}^{p}, q\right)$-Carleson measures. Some of them will be used to prove those stated in Section 1.1.

Let us start with the case $p=q$. The $p$-Carleson measures for the space $\mathcal{D}_{\alpha}^{p}$ will be called simply $\mathcal{D}_{\alpha}^{p}$-Carleson measures and have been characterized for all the admissible pairs $(p, \alpha)$ except for those with $p>2$ and $\alpha=p-1$ (see [5], 24, 31] and [34]). Let us mention that $\mathrm{Wu}$ proved in 34 that for $0<p \leq 2$, the $\mathcal{D}_{p-1^{-}}^{p}$ Carleson measures are precisely the classical Carleson measures and conjectured that the same result was true for $p>2$. Girela and Peláez proved in 18 that this conjecture is not true, proving the following result.

Theorem D. Suppose that $2<p<\infty$. Then there exists $g \in \mathcal{H o l}(\mathbb{D})$ such that the measure $\mu_{g, p}$ on $\mathbb{D}$ given by $d \mu_{g, p}(z)=\left(1-|z|^{2}\right)^{p-1}\left|g^{\prime}(z)\right|^{p} d A(z)$ is not a $\mathcal{D}_{p-1}^{p}$-Carleson measure but is a classical Carleson measure.

We remark here that our Theorem 7 improves this. Indeed, the function $g$ constructed in Theorem 7 works to prove Theorem $\mathrm{D}$ and it satisfies the additional condition of being bounded.

If $1<p<\infty$ and $\rho$ is a positive Borel measurable weight function on $\mathbb{D}$, the weighted Besov spaces $B_{p}(\rho)$ consist of those $f \in \mathcal{H}$ ol $(\mathbb{D})$ such that

$$
\|f\|_{B_{p}(\rho)} \stackrel{\text { def }}{=}|f(0)|^{p}+\int_{\mathbb{D}}\left|\left(1-|z|^{2}\right) f^{\prime}(z)\right|^{p} \rho(z) \frac{d A(z)}{\left(1-|z|^{2}\right)^{2}}<\infty .
$$

Notice that if $\rho(z) \equiv 1$, then $B_{p}(\rho)=B^{p}$. Also,

$$
\mathcal{D}_{\alpha}^{p}=B_{p}(\rho) \text { for } \rho(z)=\left(1-|z|^{2}\right)^{\alpha-p+2}, \quad 1<p<\infty, \alpha>-1 .
$$

For $1<p \leq q<\infty$ and a certain class of " $p$-admissible weights" $\rho$, Arcozzi, Rochberg and Sawyer [5] characterized the $\left(B_{p}(\rho), q\right)$-Carleson measures. The weight $\rho(z)=\left(1-|z|^{2}\right)^{\beta}$ is $p$-admissible if and only if $1-p<\beta<1$ [5, p. 445]. Using this, (1.3) and Theorem 1 of 5 we obtain the following.

Theorem E. Suppose that $1<p<\infty,-1<\alpha<p-1$ and $\mu$ is a positive Borel measure on $\mathbb{D}$. Then $\mu$ is a $\mathcal{D}_{\alpha}^{p}$-Carleson measure if and only if there exists a positive constant $C(\mu)$ such that for all $a \in \mathbb{D}$,

$$
\int_{\tilde{S}(a)}(1-|z|)^{-[\alpha-p+2] \frac{p^{\prime}}{p}-2} \mu(S(z) \cap S(a))^{p^{\prime}} d A(z) \leq C(\mu) \mu(S(a)) .
$$

The weight $\rho(z)=1-|z|$ is not $p$-admissible, and then Theorem 1 of $[5$ does not yield a characterization of the $\left(\mathcal{D}_{p-1}^{p}, q\right)$-Carleson measures $(1<p \leq q<\infty)$. In spite of this, it is natural to ask whether Theorem E remains true for $\alpha=p-1$. That is, we can formulate the following question.

Question 1. Suppose that $1<p<\infty$ and $\mu$ is a positive Borel measure on $\mathbb{D}$. Are the following two conditions equivalent?

(i) $\mu$ is a $\mathcal{D}_{p-1}^{p}$-Carleson measure.

(ii) There exists a positive constant $C(\mu)$ such that for all $a \in \mathbb{D}$,

$$
\int_{\tilde{S}(a)}(1-|z|)^{-\frac{p^{\prime}}{p}-2} \mu(S(z) \cap S(a))^{p^{\prime}} d A(z) \leq C(\mu) \mu(S(a)) .
$$


Since the weight $\rho(z)=1-|z|$ is regular in the sense of [5], an examination of the proof of Theorem $\mathrm{E}$ shows that (ii) implies (i). Next we show that the other implication is not true.

Theorem 9. Suppose that $1<p<\infty$. Then there exists a positive Borel measure $\mu$ on $\mathbb{D}$ which is a $\mathcal{D}_{p-1}^{p}$-Carleson measure for which

$$
\sup _{a \in \mathbb{D}} \frac{\int_{\tilde{S}(a)}(1-|z|)^{-\frac{p^{\prime}}{p}-2} \mu(S(z) \cap S(a))^{p^{\prime}} d A(z)}{\mu(S(a))}=\infty .
$$

Let us now turn to the upper triangle case $0<q<p$. Let $\varrho$ denote the pseudohyperbolic distance in $\mathbb{D}$,

$$
\varrho(z, w)=\left|\varphi_{w}(z)\right|=\left|\frac{z-w}{1-\bar{w} z}\right|, \quad z, w \in \mathbb{D} .
$$

The pseudohyperbolic disc of center $a$ and radius $r(a \in \mathbb{D}, 0<r<1)$ is the set $\Delta(a, r)=\{z \in \mathbb{D}: \varrho(a, z)<r\}$.

Arcozzi [6] has recently proved the following result.

Theorem F. If $1<q<p<\infty$ and $\rho$ is a $p$-admissible weight, then a positive Borel measure $\mu$ on $\mathbb{D}$ is a $\left(B_{p}(\rho), q\right)$-Carleson measure if and only if

$$
\int_{\mathbb{D}}\left(W_{\rho, p}(\mu)(z)\right)^{\frac{q(p-1)}{p-q}} d \mu(z)<\infty .
$$

Here and throughout the paper, $W_{\rho, p}(\mu)(z)$ denotes the "boundary Wolff potential associated to $\rho, p$ and $\mu "$, which is defined by

$$
W_{\rho, p}(\mu)(z)=\int_{[0, z]}\left(\frac{\mu(S(w))}{\rho(w)}\right)^{p^{\prime}-1} \frac{|d w|}{1-|w|^{2}} .
$$

Theorem $\mathbb{E}$ with $\rho(z)=\left(1-|z|^{2}\right)^{\alpha-p+2}$ yields a characterization of the $\left(\mathcal{D}_{\alpha}^{p}, q\right)$ Carleson measures for $1<q<p$ and $-1<\alpha<p-1$. On the other hand, the following characterization of the $\left(\mathcal{D}_{\alpha}^{p}, q\right)$-Carleson measures for $1<q<p$ and $\alpha>p-1$ follows from results of Luecking [25, 27], (see also [35, Theorem D]).

Theorem G. Suppose that $0<q<p, \alpha>p-1$ and $\mu$ is a positive Borel measure on $\mathbb{D}$. Then $\mu$ is a q-Carleson measure for $\mathcal{D}_{\alpha}^{p}$ if and only if

$$
\int_{\mathbb{D}}\left(\frac{\mu(\Delta(z, \delta))}{\left(1-|z|^{2}\right)^{2+\alpha}}\right)^{\frac{p}{p-q}}\left(1-|z|^{2}\right)^{\frac{p q}{p-q}+\alpha} d A(z)<\infty,
$$

for a certain $\delta \in(0,1)$.

It is natural to ask whether or not either Theorem $\mathrm{F}$ or Theorem $\mathrm{G}$ can be extended to cover the remaining case $\alpha=p-1$. First of all, an examination of the proof of Theorem $\mathrm{F}$ in [6] shows that condition (1.7) implies that $\mu$ is a Carleson measure for $\left(B_{p}(\rho), q\right)$ if we only assume that $\rho$ is regular (as defined in [5, p. 445] or in [6, p. 2]), not necessarily $p$-admissible. Since the weight $\rho(z)=1-|z|$ is regular we deduce the following.

Theorem H. If $1<q<p$ and $\mu$ is a positive finite Borel measure on $\mathbb{D}$ such that

$$
\int_{\mathbb{D}}\left(W_{1-|w|, p}(\mu)(z)\right)^{\frac{q(p-1)}{p-q}} d \mu(z)<\infty,
$$

then $\mu$ is a q-Carleson measure for $\mathcal{D}_{p-1}^{p}$. 
Thus, whenever $1<q<p$, (1.7) with $\rho(z)=1-|z|$ is a sufficient condition for $\mu$ being a $\left(\mathcal{D}_{p-1}^{p}, q\right)$-Carleson measure. Our next result asserts that it is not a necessary condition.

Theorem 10. Suppose that $1<q<p$. Then there exists a positive Borel measure $\mu$ on $\mathbb{D}$ such that $\mathcal{D}_{p-1}^{p} \subset L^{q}(d \mu)$ and

$$
\int_{\mathbb{D}}\left(W_{1-|w|, p}(\mu)(z)\right)^{\frac{q(p-1)}{p-q}} d \mu(z)=\infty .
$$

Regarding Theorem $\mathrm{G}$ we shall prove the following.

Theorem 11. Suppose that $0<q<p$ and $p>2$. Then there exists a positive Borel measure $\mu$ on $\mathbb{D}$ which is a q-Carleson measure for $H^{p}$, satisfies (1.8) with $\alpha=p-1$, but is not a $q$-Carleson measure for $\mathcal{D}_{p-1}^{p}$.

It is worth noticing that for $0<p<q$ the $\left(H^{p}, q\right)$-Carleson measures and the $\left(\mathcal{D}_{p-1}^{p}, q\right)$-Carleson measures coincide (see [11] and [19, Theorem 1]). Theorem [11 shows that this does not remain true in the upper triangle case.

Next we show that if $0<q<p$ and $\alpha>-1$, (1.8) is a necessary condition for $\mu$ being a $\left(\mathcal{D}_{\alpha}^{p}, q\right)$-Carleson measure, a result which will be used in Section 4 .

Theorem 12. Suppose that $0<q<p$ and $\alpha>-1$. If $\mu$ is a positive Borel measure on $\mathbb{D}$ which is a $\left(\mathcal{D}_{\alpha}^{p}, q\right)$-Carleson measure, then there exists $\delta_{0}>0$ such that, whenever $0<\delta<\delta_{0}$, we have

$$
\int_{\mathbb{D}}\left(\frac{\mu(\Delta(z, \delta))}{\left(1-|z|^{2}\right)^{2+\alpha}}\right)^{\frac{p}{p-q}}\left(1-|z|^{2}\right)^{\frac{p q}{p-q}+\alpha} d A(z)<\infty .
$$

We close this section by saying that from now on the paper is organized as follows:

- In Section 2 we prove Theorems 9, 10 and 11, constructing explicit examples of measures which show the impossibility of extending to the case $\alpha=p-1$ distinct results on $\left(\mathcal{D}_{\alpha}^{p}, q\right)$-Carleson measures valid for other values of $\alpha$.

- Section 3 is devoted to proving Theorem [12. In order to do so, we prove a decomposition theorem for the spaces $\mathcal{D}_{\alpha}^{p}$ which is related to one of Rochberg [30] for Bergman spaces.

- Section 4 contains proofs of our main results about the boundedness of integration operators and multipliers from $\mathcal{D}_{\alpha}^{p}$ to $\mathcal{D}_{\beta}^{q}$ in the case $q<p$ (Theorems 1 and 2). Theorem 12 is a key ingredient in our work in this section.

- Section 5 contains a proof of Theorem $[5$ and also a proof of Theorem 2 which makes no use of Theorem 12. Instead, we use a number of results concerning power series with Hadamard gaps, the sets of zeros of functions in the Dirichlet spaces and, also, arguments involving the use of Rademacher functions.

- Section 6 contains the proofs of our results concerning the characterization of the power series with Hadamard gaps which are multipliers between Dirichlet spaces (Theorems 3, 4, 6) and 7).

- Finally, Section 7 is devoted to proving Theorem 8 on compact multipliers.

\section{Counterexamples related to $\left(\mathcal{D}_{p-1}^{p}, q\right)$-Carleson measures}

This section is devoted to constructing the measures $\mu$ whose existence is insured in Theorems 9, 10 and 11. These measures are radial; in fact, they are all of the 
form

$$
d \mu(z)=\frac{d A(z)}{(1-|z|) \log ^{\gamma} \frac{e}{1-|z|}}
$$

for an appropriate $\gamma>0$. We shall list a number of results about these measures in Proposition 1, but before doing so we need to introduce some further notation.

For $a \in \mathbb{D}$ we define

$$
\hat{S}(a)=\left\{z \in \mathbb{D}: 1-|z| \leq \frac{1}{2}(1-|a|),\left|\frac{\arg (a \bar{z})}{2 \pi}\right| \leq \frac{1-|a|}{4}\right\} .
$$

It is clear that

$$
z \in \hat{S}(a) \Rightarrow S(z) \subset S(a) .
$$

Proposition 1. Suppose that $\gamma>1$ and let $\mu$ be the Borel measure on $\mathbb{D}$ defined by (2.1). Then:

(i) $\mu(S(a)) \asymp \mu(\hat{S}(a)) \asymp(1-|a|) \log ^{1-\gamma} \frac{e}{1-|a|}$.

(ii) If $0<q \leq p \leq 2$, then $\mu$ is $\left(\mathcal{D}_{p-1}^{p}, q\right)$-Carleson measure.

(iii) If $p>2,0<q \leq p$ and $\gamma>1+\frac{q}{2}$, then $\mu$ is $\left(\mathcal{D}_{p-1}^{p}, q\right)$-Carleson measure.

Proof. A simple computation gives (i).

Suppose that $0<q \leq p \leq 2$. Take $f \in \mathcal{D}_{p-1}^{p}$. Since $\mathcal{D}_{p-1}^{p} \subset H^{p}$ and $M_{s}(r, f)$ increases with $s$, we have

$$
\begin{aligned}
\int_{\mathbb{D}}|f(z)|^{q} d \mu(z) & \leq \int_{0}^{1} M_{q}^{q}(r, f) \frac{d r}{(1-r) \log ^{\gamma} \frac{e}{1-r}} \\
& \leq \int_{0}^{1} M_{p}^{q}(r, f) \frac{d r}{(1-r) \log ^{\gamma} \frac{e}{1-r}} \\
& \leq C\|f\|_{H^{p}}^{q} \int_{0}^{1} \frac{d r}{(1-r) \log ^{\gamma} \frac{e}{1-r}}<\infty .
\end{aligned}
$$

Thus, we have proved that $\mathcal{D}_{p-1}^{p} \subset L^{q}(d \mu)$. Hence, (ii) holds.

Suppose now that $p>2,0<q \leq p$ and $\gamma>1+\frac{q}{2}$ and take $f \in \mathcal{D}_{p-1}^{p}$. We have $M_{p}\left(r, f^{\prime}\right)=\mathrm{O}\left(\frac{1}{1-r}\right)$, as $r \rightarrow 1$, and then, using Theorem 1.1 of [17], we deduce that

which implies

$$
M_{p}(r, f)=\mathrm{O}\left(\left(\log \frac{1}{1-r}\right)^{1 / 2}\right), \quad \text { as } r \rightarrow 1,
$$

$$
\begin{aligned}
\int_{\mathbb{D}}|f(z)|^{q} d \mu(z) & \leq \int_{0}^{1} M_{q}^{q}(r, f) \frac{d r}{(1-r)\left(\log \frac{e}{1-r}\right)^{\gamma}} \\
& \leq \int_{0}^{1} M_{p}^{q}(r, f) \frac{d r}{(1-r)\left(\log \frac{e}{1-r}\right)^{\gamma}} \\
& \leq C \int_{0}^{1} \frac{d r}{(1-r)\left(\log \frac{e}{1-r}\right)^{\gamma-\frac{q}{2}}}<\infty,
\end{aligned}
$$

that is, $f \in L^{q}(d \mu)$. Hence, we have proved (iii). 
Proof of Theorem 9. We split the proof into two cases.

Case 1: $1<p \leq 2$. Take $\mu$ defined as in (2.1) for a certain $\gamma$ with $(\gamma-1)\left(p^{\prime}-1\right)<$ $1<(\gamma-1) p^{\prime}$. Using Proposition 1(ii) we deduce that $\mu$ is a $\mathcal{D}_{p-1}^{p}$-Carleson measure. On the other hand, (2.2) and Proposition 1 (i) imply

$$
\begin{aligned}
& \int_{\tilde{S}(a)}(1-|z|)^{-\frac{p^{\prime}}{p}-2} \mu(S(z) \cap S(a))^{p^{\prime}} d A(z) \\
\geq & \int_{\hat{S}(a)}(1-|z|)^{-\frac{p^{\prime}}{p}-2} \mu(S(z))^{p^{\prime}} d A(z) \\
\geq & C \int_{\hat{S}(a)}(1-|z|)^{-1}\left(\log \frac{e}{1-|z|}\right)^{-p^{\prime}(\gamma-1)} d A(z) \\
\asymp & (1-|a|)\left(\log \frac{e}{1-|a|}\right)^{1-p^{\prime}(\gamma-1)} .
\end{aligned}
$$

This, the fact that $(\gamma-1)\left(p^{\prime}-1\right)<1$ and Proposition 1 (i) yield (1.6).

Case 2: $2<p<\infty$. Take $\mu$ defined as in (2.1) with $1+\frac{p}{2}<\gamma<p$. By Proposition 1 (iii), $\mu$ is a $\mathcal{D}_{p-1}^{p}$-Carleson measure. Arguing as in case 1 we deduce that

$$
\int_{\tilde{S}(a)}(1-|z|)^{-\frac{p^{\prime}}{p}-2} \mu(S(z) \cap S(a))^{p^{\prime}} d A(z) \geq(1-|a|) \log \left(\frac{e}{1-|a|}\right)^{1-p^{\prime}(\gamma-1)} .
$$

Now, the condition $\gamma<p$ implies that $1-p^{\prime}(\gamma-1)>1-\gamma$ and then, using Proposition 1(i), (1.6) follows.

Proof of Theorem 10. The proof is split into two cases.

Case 1: $1<p \leq 2$. Take $\gamma$ such that $1<\gamma \leq 1+\frac{q(p-1)}{p}$ and let $\mu$ be defined as in (2.1) with this $\gamma$. Proposition 1 (ii) shows that $\mu$ is a $\left(\mathcal{D}_{p-1}^{p}, q\right)$-Carleson measure.

On the other hand, Proposition 1 (i) implies that if $w \in \mathbb{D}$,

$$
\mu(S(w)) \asymp \frac{1-|w|}{\log ^{\gamma-1} \frac{e}{1-|w|}} .
$$

Then, bearing in mind the election of $\gamma$, if $|z| \geq \frac{1}{2}$, we have

$$
\begin{aligned}
W_{(1-|w|), p}(\mu)(z) & \geq C \int_{0}^{|z|}\left(\log \frac{e}{1-|w|}\right)^{\frac{-\gamma+1}{p-1}} \frac{|d w|}{1-|w|} \\
& \geq C\left(\log \frac{e}{1-|z|}\right)^{\frac{p-\gamma}{p-1}} .
\end{aligned}
$$

This and the fact that $\gamma+\frac{q(\gamma-p)}{p-q}<1$ imply

$$
\begin{aligned}
& \int_{\mathbb{D}}\left(W_{1-|w|, p}(\mu)(z)\right)^{\frac{q(p-1)}{p-q}} d \mu(z) \\
\geq & C \int_{\frac{1}{2}}^{1}\left[\left(\log \frac{e}{1-r}\right)^{\frac{p-\gamma}{p-1}}\right]^{\frac{q(p-1)}{p-q}} \frac{d r}{(1-r) \log ^{\gamma} \frac{e}{1-r}} \\
\geq & C \int_{\frac{1}{2}}^{1} \frac{d r}{(1-r)\left(\log \frac{e}{1-r}\right)^{\gamma+\frac{q(\gamma-p)}{p-q}}}=\infty .
\end{aligned}
$$


This finishes the proof in this case.

Case 2: $2<p<\infty$. Take $\varepsilon$ such that $0<\varepsilon<q\left(\frac{1}{2}-\frac{1}{p}\right)$. Notice that then

$$
\frac{q}{2}+\varepsilon<p-1 \text { and } \frac{p \varepsilon+q\left(-\frac{p}{2}+1\right)}{p-q}<0 .
$$

Let $\mu$ be the measure defined by (2.1) with $\gamma=1+\frac{q}{2}+\varepsilon$, that is,

$$
d \mu(z)=\frac{d A(z)}{(1-|z|)\left(\log \frac{e}{1-|z|}\right)^{1+\frac{q}{2}+\varepsilon}} .
$$

Using Proposition 1 we deduce that $\mu$ is a $\left(\mathcal{D}_{p-1}^{p}, q\right)$-Carleson measure and that

$$
\mu(S(w)) \geq C \frac{1-|w|}{\left(\log \frac{e}{1-|w|}\right)^{\frac{q}{2}+\varepsilon}},
$$

which, bearing in mind the election of $\varepsilon$, implies that if $|z| \geq \frac{1}{2}$, then

$$
W_{(1-|w|), p}(\mu)(z) \geq C\left(\log \frac{e}{1-|z|}\right)^{\frac{p-1-q / 2-\varepsilon}{p-1}}
$$

This yields

$$
\begin{aligned}
& \int_{\mathbb{D}}\left(W_{1-|w|, p}(\mu)(z)\right)^{\frac{q(p-1)}{p-q}} d \mu(z) \\
& \geq C \int_{\frac{1}{2}}^{1}\left[\left(\log \frac{e}{1-r}\right)^{\frac{p-1-q / 2-\varepsilon}{p-1}}\right]^{\frac{q(p-1)}{p-q}} \frac{d r}{(1-r)\left(\log \frac{e}{1-r}\right)^{1+\frac{q}{2}+\varepsilon}} \\
& \geq C \int_{\frac{1}{2}}^{1} \frac{d r}{(1-r)\left(\log \frac{e}{1-r}\right)^{1+\frac{p \varepsilon+q\left(-\frac{p}{2}+1\right)}{p-q}}}=\infty
\end{aligned}
$$

This finishes the proof.

Proof of Theorem 11. Take $\beta$ and $\gamma$ such that $\frac{1}{p}<\beta<\frac{1}{2}$ and $1<\gamma \leq 1+q\left(\frac{1}{2}-\beta\right)$ and let $\mu$ be the measure defined by

$$
d \mu(z)=\frac{d A(z)}{(1-|z|) \log ^{\gamma}\left(\frac{e}{1-|z|}\right)} .
$$

Since $\mu$ is a finite radial measure and $H^{p} \subset H^{q}$, it follows readily that $H^{p} \subset$ $L^{q}(d \mu)$. Also, it is easy to see that there exist positive constants $\alpha, \beta$ (depending on $\delta$ ) such that, for every $z$, the pseudohyperbolic disc $\Delta(z, \delta)$ is contained in the ring $\{w: \alpha(1-|z|)<1-|w|<\beta(1-|z|)\}$. This implies that

$$
\mu(\Delta(z, \delta)) \leq \frac{C(1-|z|)}{\log ^{\gamma}\left(\frac{e}{1-|z|}\right)}
$$


and then it follows that

$$
\begin{aligned}
& \int_{\mathbb{D}}\left(\frac{\mu(\Delta(z, \delta))}{\left(1-|z|^{2}\right)^{p+1}}\right)^{\frac{p}{p-q}}\left(1-|z|^{2}\right)^{\frac{p q}{p-q}+p-1} d A(z) \\
& \leq C \int_{\mathbb{D}}\left(\left(1-|z|^{2}\right)^{-p} \log ^{-\gamma} \frac{e}{1-|z|^{2}}\right)^{\frac{p}{p-q}}\left(1-|z|^{2}\right)^{\frac{p q}{p-q}+p-1} d A(z) \\
& \leq C \int_{0}^{1}\left(1-r^{2}\right)^{-1}\left(\log \frac{e}{1-r^{2}}\right)^{-\gamma \frac{p}{p-q}} d r<\infty,
\end{aligned}
$$

where in the last inequality we have used that $\gamma \frac{p}{p-q}>1$.

Next, we shall see that $\mathcal{D}_{p-1}^{p} \not \subset L^{q}(d \mu)$. Set

$$
f(z)=\sum_{k=0}^{\infty} \frac{1}{(k+1)^{\beta}} z^{2^{k}}=\sum_{k=0}^{\infty} a_{n} z^{n}, \quad z \in \mathbb{D} .
$$

Since $p \beta>1$, using Proposition $\mathrm{A}$, we see that $f \in \mathcal{D}_{p-1}^{p}$. Observe that

$$
\sum_{n=0}^{N}\left|a_{n}\right|^{2} \approx(\log N)^{1-2 \beta}, \quad \text { as } N \rightarrow \infty
$$

which implies that

$$
M_{2}(r, f) \approx\left(\log \frac{1}{1-r}\right)^{1 / 2-\beta}, \quad \text { as } r \rightarrow 1 .
$$

Moreover, since $f$ is given by a power series with Hadamard gaps,

$$
M_{2}(r, f) \approx M_{q}(r, f) \text { for all } q \in(0, \infty),
$$

consequently, bearing in mind the election of $\beta$ and $\gamma$, we deduce that

$$
\begin{aligned}
\int_{\mathbb{D}}|f(z)|^{q} d \mu(z) & =\int_{0}^{1} I_{q}(r, f)(1-r)^{-1} \log ^{-\gamma} \frac{e}{1-r} d r \\
& \geq C \int_{0}^{1} M_{2}^{q}(r, f)(1-r)^{-1} \log ^{-\gamma} \frac{e}{1-r} d r \\
& \geq C \int_{0}^{1}(1-r)^{-1}\left(\log \frac{e}{1-r}\right)^{-\gamma+q\left(\frac{1}{2}-\beta\right)} d r=\infty .
\end{aligned}
$$

Hence, $f \notin L^{q}(d \mu)$. This finishes the proof.

\section{A necessary condition for $\mu$ Being a $\left(\mathcal{D}_{\alpha}^{p}, q\right)$-Carleson measure}

$$
(0<q<p, \alpha>-1)
$$

Our aim in this section is to give a proof of Theorem 12. A key ingredient in our work will be a decomposition theorem for $\mathcal{D}_{\alpha}^{p}$.

3.1. A decomposition theorem for the $\mathcal{D}_{\alpha}^{p}$-spaces. A sequence $\left\{z_{k}\right\}_{k=1}^{\infty} \subset \mathbb{D}$ is said to be uniformly discrete if it is separated in the pseudohyperbolic metric, that is, if there exists a positive constant $\gamma$ such that

$$
\varrho\left(z_{j}, z_{k}\right) \geq \gamma, \quad k=1,2, \ldots
$$

For $0<\varepsilon<1$, a sequence $\left\{z_{k}\right\}_{k=1}^{\infty} \subset \mathbb{D}$ is said to be an " $\varepsilon$-net" if $\mathbb{D}=$ $\bigcup_{k=1}^{\infty} \Delta\left(z_{k}, \varepsilon\right)$. 
A sequence $\left\{z_{k}\right\}_{k=1}^{\infty} \subset \mathbb{D}$ is said to be a $\delta$-lattice if it is separated with constant $\delta / 5$ and is a $5 \delta$-net.

Now we can state the above-mentioned decomposition theorem for $\mathcal{D}_{\alpha}^{p}$. It is based on that of R. Rochberg for Bergman spaces 30, Theorem 2.2]. A similar result was proved by $\mathrm{Z}$. Wu and L. Yang in [33. We remark also that many of the cases of our theorem (in the more general context of several complex variables) are contained in the work of Peloso [29] (see also [9] and 32]). We shall include a proof for the sake of completeness.

Theorem 13. Suppose that $0<p<\infty, \alpha>-1$ and $a>(2+\alpha) \max \left\{1, \frac{1}{p}\right\}$. Then there exists a positive constant $\delta_{0}$ such that for any $\delta \in\left(0, \delta_{0}\right)$ and any $\delta$ lattice $\left\{z_{j}\right\}_{j=1}^{\infty} \subset \mathbb{D} \backslash\{0\}$, there exists a positive constant $C=C(\alpha, p, a, \delta)$ such that:

(i) If $f \in \mathcal{D}_{\alpha}^{p}$, then there exists a sequence $\left\{a_{j}\right\}_{j=1}^{\infty} \in l^{p}$ and a constant $M=$ $M\left(\left\{z_{j}\right\},\left\{a_{j}\right\}, f(0)\right)$ so that

$$
f(z)=M+\sum_{j=1}^{\infty} \frac{a_{j}\left(1-\left|z_{j}\right|^{2}\right)^{a-\frac{2+\alpha}{p}}}{\overline{z_{j}}\left(1-\overline{z_{j}} z\right)^{a-1}}
$$

and $\left\|\left\{a_{j}\right\}_{j=1}^{\infty}\right\|_{l^{p}} \leq C\|f(z)-f(0)\|_{\mathcal{D}_{\alpha}^{p}}$.

(ii) If $\left\{a_{j}\right\}_{j=1}^{\infty} \in l^{p}$, then the function $f$ defined by (3.1) converges uniformly on compact subsets of $\mathbb{D}$ to an analytic function $f \in \mathcal{D}_{\alpha}^{p}$ such that

$$
\|f(z)-f(0)\|_{\mathcal{D}_{\alpha}^{p}} \leq C\left\|\left\{a_{j}\right\}_{j=1}^{\infty}\right\|_{l^{p}} .
$$

Proof. Let $\delta_{0}$ be the same constant that appears in the statement of Theorem 2.2 of 30 .

Suppose that $f \in \mathcal{D}_{\alpha}^{p}$, that is, $f^{\prime} \in A_{\alpha}^{p}$. Then by Theorem 2.2 of 30 for any $\delta$-lattice in $\left\{z_{j}\right\} \subset \mathbb{D} \backslash\{0\}$ there is a constant $C=C(\alpha, p, a, \delta)$ and a sequence $\left\{b_{j}\right\}_{j=1}^{\infty} \in l^{p}$ so that

$$
f^{\prime}(z)=\sum_{j=1}^{\infty} \frac{b_{j}\left(1-\left|z_{j}\right|^{2}\right)^{a-\frac{2+\alpha}{p}}}{\left(1-\overline{z_{j}} z\right)^{a}}
$$

with

$$
\left\|\left\{b_{j}\right\}_{j=1}^{\infty}\right\|_{l^{p}} \leq C|| f^{\prime}\left\|_{A_{\alpha}^{p}}=\right\| f(z)-f(0) \|_{\mathcal{D}_{\alpha}^{p}} .
$$

We claim that

$$
\sum_{j=1}^{\infty}\left|\frac{b_{j}\left(1-\left|z_{j}\right|^{2}\right)^{a-\frac{2+\alpha}{p}}}{\overline{z_{j}}}\right|<\infty .
$$

If $p \leq 1$ this is clear because $a-\frac{2+\alpha}{p}>0, \lim _{j \rightarrow \infty}\left|z_{j}\right|=1$, and the fact that $l^{p} \subset l^{1}$. If $p>1$, using Hölder's inequality, the facts that $\frac{p}{p-1}\left(a-\frac{2+\alpha}{p}\right)=\frac{p a-(2+\alpha)}{p-1}>2+\alpha$ and $\sum_{j=1}^{\infty}\left(1-\left|z_{j}\right|^{2}\right)^{1+\eta}<\infty$ for every $\eta>0$ (see, e.g., [14, Theorem 1] or [13, p. 68]), 
we have

$$
\begin{aligned}
& \sum_{j=1}^{\infty}\left|\frac{b_{j}\left(1-\left|z_{j}\right|^{2}\right)^{a-\frac{2+\alpha}{p}}}{\overline{z_{j}}}\right| \\
\leq & \left(\sum_{j=1}^{\infty}\left|\frac{b_{j}}{\overline{z_{j}}}\right|^{p}\right)^{1 / p}\left(\sum_{j=1}^{\infty}\left(1-\left|z_{j}\right|^{2}\right)^{\frac{p}{p-1}\left(a-\frac{2+\alpha}{p}\right)}\right)^{1-\frac{1}{p}} \\
\leq & \left(\sum_{j=1}^{\infty}\left|\frac{b_{j}}{\overline{z_{j}}}\right|^{p}\right)^{1 / p}\left(\sum_{j=1}^{\infty}\left(1-\left|z_{j}\right|^{2}\right)^{2+\alpha}\right)^{1-\frac{1}{p}}<\infty .
\end{aligned}
$$

Once (3.4) has been proved, we can write

$$
\begin{aligned}
& f(z)=f(0)+\int_{0}^{z} f^{\prime}(\xi) d \xi \\
& =f(0)-\frac{1}{a-1} \sum_{j=1}^{\infty} \frac{b_{j}\left(1-\left|z_{j}\right|^{2}\right)^{a-\frac{2+\alpha}{p}}}{\overline{z_{j}}}+\frac{1}{a-1} \sum_{j=1}^{\infty} \frac{b_{j}\left(1-\left|z_{j}\right|^{2}\right)^{a-\frac{2+\alpha}{p}}}{\overline{z_{j}}\left(1-\overline{z_{j}} z\right)^{a-1}} .
\end{aligned}
$$

Hence (i) is proved.

Now we turn to prove (ii). Suppose that $\left\{a_{j}\right\}_{j=1}^{\infty} \in l^{p}$ and $f$ is defined by (3.1). Then arguing as above we have that $f \in \mathcal{H}$ ol $(\mathbb{D})$ and it follows that

$$
f^{\prime}(z)=(a-1) \sum_{j=1}^{\infty} \frac{a_{j}\left(1-\left|z_{j}\right|^{2}\right)^{a-\frac{2+\alpha}{p}}}{\left(1-\overline{z_{j}} z\right)^{a}} .
$$

Then, bearing in mind that $a>(2+\alpha) \max \left\{1, \frac{1}{p}\right\}$ and Theorem 2.2 of [30], we have that

$$
\|f(z)-f(0)\|_{\mathcal{D}_{\alpha}^{p}}=\left\|f^{\prime}\right\|_{A_{\alpha}^{p}} \leq C||\left\{a_{j}\right\}_{j=1}^{\infty} \|_{l^{p}}
$$

3.2. A proof of Theorem 12, Our proof of Theorem 12 will also use the Rademacher functions $\left\{r_{j}(t)\right\}_{j=0}^{\infty}$ defined by

$$
\begin{array}{r}
r_{0}(t)= \begin{cases}1, & \text { if } 0<t<1 / 2, \\
-1, & \text { if } 1 / 2<t<1, \\
0, & \text { if } t=0,1 / 2,1,\end{cases} \\
r_{n}(t)=r_{0}\left(2^{n} t\right), \quad n=1,2, \ldots
\end{array}
$$

See, e.g., [38, Chapter V, Vol. I] or [12, Appendix A] for the properties of these functions. In particular, we shall use Khinchine's inequality, which we state as follows.

Proposition B (Khinchine's inequality). If $\left\{c_{k}\right\}_{k=1}^{\infty} \in \ell^{2}$, then the series $\sum_{k=1}^{\infty} c_{k} r_{k}(t)$ converges almost everywhere. Furthermore, for $0<p<\infty$ there exist positive constants $A_{p}, B_{p}$ such that for every sequence $\left\{c_{k}\right\}_{k=1}^{\infty} \in \ell^{2}$ we have

$$
A_{p}\left(\sum_{k=1}^{\infty}\left|c_{k}\right|^{2}\right)^{p / 2} \leq \int_{0}^{1}\left|\sum_{k=1}^{\infty} c_{k} r_{k}(t)\right|^{p} d t \leq B_{p}\left(\sum_{k=1}^{\infty}\left|c_{k}\right|^{2}\right)^{p / 2} .
$$


Proof of Theorem 12, Assume that $\mathcal{D}_{\alpha}^{p} \subset L^{q}(d \mu)$. Then, by the closed graph theorem, there exists a positive constant $C$ such that $\|f\|_{L^{q}(d \mu)} \leq C\|f\|_{\mathcal{D}_{\alpha}^{p}}$, for all $f \in \mathcal{D}_{\alpha}^{p}$.

Take $a>(2+\alpha) \max \left\{1, \frac{1}{p}\right\}$ and let $\delta_{0}$ be the constant which appears in the statement of Theorem 13. Take $\delta$ with $0<\delta<\delta_{0}$ and let $\left\{z_{j}\right\}_{j=0}^{\infty} \subset \mathbb{D} \backslash\{0\}$ be a $\delta$-lattice. Let $\left\{b_{j}\right\}_{j=0}^{\infty}$ be an arbitrary $\ell^{p}$ sequence. Using Theorem 13 , we see that if

$$
f(z)=\sum_{j=0}^{\infty} \frac{b_{j}\left(1-\left|z_{j}\right|^{2}\right)^{a-\frac{2+\alpha}{p}}}{\left(1-\overline{z_{j}} z\right)^{a-1}}
$$

then $f \in \mathcal{D}_{\alpha}^{p}$ and $\|f\|_{\mathcal{D}_{\alpha}^{p}} \asymp\left\|\left\{b_{j}\right\}\right\|_{\ell^{p}}$.

Let $\left\{r_{j}(t)\right\}_{j=0}^{\infty}$ be the Rademacher functions and let

$$
f_{t}(z)=\sum_{j=0}^{\infty} r_{j}(t) \frac{b_{j}\left(1-\left|z_{j}\right|^{2}\right)^{a-\frac{2+\alpha}{p}}}{\left(1-\overline{z_{j}} z\right)^{a-1}} .
$$

By Theorem 13 ,

$$
\left\|f_{t}\right\|_{\mathcal{D}_{\alpha}^{p}} \approx\|f\|_{\mathcal{D}_{\alpha}^{p}} \approx\left\|\left\{b_{j}\right\}_{j=0}^{\infty}\right\|_{l^{p}}
$$

and then it follows that

$$
\left\|f_{t}\right\|_{L^{q}(d \mu)}^{q} \leq C\|f\|_{\mathcal{D}_{\alpha}^{p}}^{q} \approx C\left\|\left\{b_{j}\right\}_{j=0}^{\infty}\right\|_{l^{p}}^{q},
$$

which, together with Fubini's theorem, Khinchine's inequality and the well-known fact that

$$
\left(1-|z|^{2}\right)^{2} \asymp|1-\bar{\xi} z|^{2} \asymp\left(1-|\xi|^{2}\right)^{2} \asymp A(\Delta(\xi, \delta)), z \in \Delta(\xi, \delta),
$$

with constants independent of $\xi$, gives

$$
\begin{aligned}
& \left\|\left\{b_{j}\right\}_{j=0}^{\infty}||_{l^{p}}^{q}=\int_{0}^{1}\right\|\left\{b_{j}\right\}_{j=0}^{\infty} \|_{l^{p}}^{q} d t \\
& \geq C \int_{0}^{1}\left\|f_{t}\right\|_{L^{q}(d \mu)}^{q} d t \\
& \geq C \int_{\mathbb{D}}\left(\sum_{j=0}^{\infty} \frac{\left|b_{j}\right|^{2}\left(1-\left|z_{j}\right|^{2}\right)^{2\left(a-\frac{2+\alpha}{p}\right)}}{\left|1-\overline{z_{j}} z\right|^{2(a-1)}}\right)^{\frac{q}{2}} d \mu(z) \\
& \geq C \sum_{n=0}^{\infty} \int_{\Delta\left(z_{n}, \delta\right)}\left(\sum_{j=0}^{\infty} \frac{\left|b_{j}\right|^{2}\left(1-\left|z_{j}\right|^{2}\right)^{2\left(a-\frac{2+\alpha}{p}\right)}}{\left|1-\overline{z_{j}} z\right|^{2(a-1)}}\right)^{\frac{q}{2}} d \mu(z) \\
& \geq C \sum_{n=0}^{\infty}\left|b_{n}\right|^{q}\left(1-\left|z_{n}\right|^{2}\right)^{q\left(a-\frac{2+\alpha}{p}\right)} \int_{\Delta\left(z_{n}, \delta\right)} \overline{\left|1-\overline{z_{n}} z\right|^{q(a-1)}} \\
& \geq C \sum_{n=0}^{\infty}\left|b_{n}\right|^{q}\left(1-\left|z_{n}\right|^{2}\right)^{q\left(1-\frac{2+\alpha}{p}\right)} \mu\left(\Delta\left(z_{n}, \delta\right)\right) .
\end{aligned}
$$

That is, we have proved that

$$
\sum_{n=0}^{\infty}\left|b_{n}\right|^{q}\left(1-\left|z_{n}\right|^{2}\right)^{q\left(1-\frac{2+\alpha}{p}\right)} \mu\left(\Delta\left(z_{n}, \delta\right)\right) \leq C\left(\sum_{n=0}^{\infty}\left|b_{n}\right|^{p}\right)^{\frac{q}{p}} .
$$


Since $\left\{b_{n}\right\}$ is an arbitrary $\ell^{p}$ sequence, we deduce that the sequence

$$
\left\{\left(1-\left|z_{n}\right|^{2}\right)^{q\left(1-\frac{2+\alpha}{p}\right)} \mu\left(\Delta\left(z_{n}, \delta\right)\right)\right\}
$$

belongs to the dual of $\ell^{p / q}$, which is $\ell^{p /(p-q)}$. Using this and (3.7) we deduce that

$$
\sum_{n=0}^{\infty}\left(1-\left|z_{n}\right|^{2}\right)^{q\left(1-\frac{2+\alpha}{p}\right) \frac{p}{p-q}-2} \mu\left(\Delta\left(z_{n}, \delta\right)\right)^{\frac{p}{p-q}} A\left(\Delta\left(z_{n}, \delta\right)\right)<\infty .
$$

This is a discrete version of (1.11). The continuous version can be obtained by arguing as on pp. 337-338 of [27]. This finishes the proof.

\section{Multipliers and integration operators from $\mathcal{D}_{\alpha}^{p}$ to $\mathcal{D}_{\beta}^{q}, 0<q<p$}

This section is devoted to proving our results on the boundedness of the operators of integration and multiplication from $\mathcal{D}_{\alpha}^{p}$ to $\mathcal{D}_{\beta}^{q}, 0<q<p$.

Proofs of Theorem 1 and Theorem 2, Using the Closed Graph Theorem, we see that $I_{g}$ maps $\mathcal{D}_{\alpha}^{p}$ to $\mathcal{D}_{\beta}^{q}$ if and only if there exists a positive constant $C$ such that

$$
\int_{\mathbb{D}}\left|g(z) f^{\prime}(z)\right|^{q}\left(1-|z|^{2}\right)^{\beta} d A(z) \leq C\|f\|_{\mathcal{D}_{\alpha}^{p}}^{q}, \quad \text { for all } f \in \mathcal{D}_{\alpha}^{p} .
$$

This is the same as saying that $M_{g}$ is bounded from $A_{\alpha}^{p}$ to $A_{\beta}^{q}$. Using part (iv) of Theorem 1 of 35 . (and its proof) we see that this happens if and only if

$$
\int_{\mathbb{D}}|g(z)|^{\frac{p q}{p-q}}\left(1-|z|^{2}\right)^{\frac{p \beta-q \alpha}{p-q}} d A(z)<\infty
$$

and this is equivalent to saying that:

$$
g \in A_{\frac{p \beta-q \alpha}{p-q}}^{\frac{p q}{p-q}} \text { if } \frac{p \beta-q \alpha}{p-q}>-1
$$

and that $g=0$ if $\frac{p \beta-q \alpha}{p-q} \geq-1$. Thus we have proved part (1) of Theorem 1 and the equivalence $(1) \Leftrightarrow(3)$ in Theorem 2 .

Next we shall prove part (2) of Theorem 1 and the equivalence $(2) \Leftrightarrow(3)$ in Theorem 2. So take $g \in \mathcal{H o l}(\mathbb{D})$ and assume that $M_{g}$ is bounded from $\mathcal{D}_{\alpha}^{p}$ to $\mathcal{D}_{\beta}^{q}$. Take $a>(2+\alpha) \max \left\{1, \frac{1}{p}\right\}$ and let $\delta_{0}$ be the constant which appears in the statement of Theorem 13. Take $\delta$ with $0<\delta<\delta_{0}$ and let $\left\{z_{j}\right\}_{j=1}^{\infty} \subset \mathbb{D} \backslash\{0\}$ be a $\delta$-lattice. Let $\left\{b_{j}\right\}_{j=1}^{\infty}$ be a sequence in $\ell^{p}$. Set

$$
a_{j}=\overline{z_{j}} b_{j}, \quad j=1,2, \ldots
$$

Clearly, $\left\{a_{j}\right\}_{j=1}^{\infty} \in \ell^{p}$ and using Theorem 13 we see that setting

$$
f(z)=\sum_{j=1}^{\infty} \frac{a_{j}\left(1-\left|z_{j}\right|^{2}\right)^{a-\frac{2+\alpha}{p}}}{\overline{z_{j}}\left(1-\overline{z_{j}} z\right)^{a-1}}=\sum_{j=1}^{\infty} \frac{b_{j}\left(1-\left|z_{j}\right|^{2}\right)^{a-\frac{2+\alpha}{p}}}{\left(1-\overline{z_{j}} z\right)^{a-1}},
$$

we have that $f \in \mathcal{D}_{\alpha}^{p}$ and $\|f\|_{\mathcal{D}_{\alpha}^{p}} \approx\left\|\left\{b_{j}\right\}_{j=0}^{\infty}\right\|_{l^{p}}$.

Let $\left\{r_{j}(t)\right\}_{j=0}^{\infty}$ be the Rademacher functions and set

$$
f_{t}(z)=\sum_{j=0}^{\infty} r_{j}(t) \frac{b_{j}\left(1-\left|z_{j}\right|^{2}\right)^{a-\frac{2+\alpha}{p}}}{\left(1-\overline{z_{j}} z\right)^{a-1}}, \quad z \in \mathbb{D}, 0 \leq t \leq 1 .
$$

Using Theorem 13 again, we deduce that

$$
\left\|f_{t}\right\|_{\mathcal{D}_{\alpha}^{p}} \approx\|f\|_{\mathcal{D}_{\alpha}^{p}} \approx\left\|\left\{b_{j}\right\}_{j=0}^{\infty}\right\|_{l^{p}}
$$


and then it follows that

$$
\left\|M_{g}\left(f_{t}\right)\right\|_{\mathcal{D}_{\beta}^{q}}^{q} \leq C\|f\|_{\mathcal{D}_{\alpha}^{p}}^{q} \approx\left\|\left\{b_{j}\right\}_{j=0}^{\infty}\right\|_{l^{p}}^{q}
$$

which, together with the fact that

$$
\left(f_{t} g\right)^{\prime}(z)=\sum_{j=0}^{\infty} r_{j}(t) \frac{b_{j}\left(1-\left|z_{j}\right|^{2}\right)^{a-\frac{2+\alpha}{p}}}{\left(1-\overline{z_{j}} z\right)^{a}}\left[(a-1) \overline{z_{j}} g(z)+g^{\prime}(z)\left(1-\overline{z_{j}} z\right)\right],
$$

Fubini's theorem, Khinchine's inequality and (3.7), gives

$$
\begin{aligned}
& \left\|\left\{b_{j}\right\}_{j=0}^{\infty}\right\|_{l^{p}}^{q}=\int_{0}^{1}\left\|\left\{b_{j}\right\}_{j=0}^{\infty}\right\|_{l^{p}}^{q} d t \\
& \geq C \int_{0}^{1}\left\|M_{g}\left(f_{t}\right)\right\|_{\mathcal{D}_{\beta}^{q}}^{q} d t \\
& \geq C \int_{\mathbb{D}}\left[\int_{0}^{1}\left|\left(f_{t} g\right)^{\prime}(z)\right|^{q} d t\right]\left(1-|z|^{2}\right)^{\beta} d A(z) \\
& \geq C \int_{\mathbb{D}}\left(\sum_{j=0}^{\infty} \frac{\left|b_{j}\right|^{2}\left(1-\left|z_{j}\right|^{2}\right)^{2\left(a-\frac{2+\alpha}{p}\right)}}{\left|1-\overline{z_{j}} z\right|^{2 a}}\left|(a-1) \overline{z_{j}} g(z)+g^{\prime}(z)\left(1-\overline{z_{j}} z\right)\right|^{2}\right)^{\frac{q}{2}}\left(1-|z|^{2}\right)^{\beta} d A(z) \\
& \geq C \sum_{n=0}^{\infty} \int_{\Delta\left(z_{n}, \delta\right)}\left(\sum_{j=0}^{\infty} \frac{\left|b_{j}\right|^{2}\left(1-\left|z_{j}\right|^{2}\right)^{2\left(a-\frac{2+\alpha}{p}\right)}\left|(a-1) \overline{z_{j}} g(z)+g^{\prime}(z)\left(1-\overline{z_{j}} z\right)\right|^{2}}{\left|1-\overline{z_{j}} z\right|^{2 a}}\right)^{\frac{q}{2}}\left(1-|z|^{2}\right)^{\beta} d A(z) \\
& \geq C \sum_{n=0}^{\infty}\left|b_{n}\right|^{q}\left(1-\left|z_{n}\right|^{2}\right)^{q\left(a-\frac{2+\alpha}{p}\right)} \int_{\Delta\left(z_{n}, \delta\right)} \frac{\left|(a-1) \overline{z_{n}} g(z)+g^{\prime}(z)\left(1-\overline{z_{n}} z\right)\right|^{q}}{\left|1-\overline{z_{n}} z\right|^{q a}}\left(1-|z|^{2}\right)^{\beta} d A(z) \\
& \geq C \sum_{n=0}^{\infty}\left|b_{n}\right|^{q}\left(1-\left|z_{n}\right|^{2}\right)^{\beta-\frac{q(2+\alpha)}{p}} \int_{\Delta\left(z_{n}, \delta\right)}\left|(a-1) \overline{z_{n}} g(z)+g^{\prime}(z)\left(1-\overline{z_{n}} z\right)\right|^{q} d A(z) .
\end{aligned}
$$

Summarizing, we have proved

$$
\sum_{n=0}^{\infty}\left|b_{n}\right|^{q}\left(1-\left|z_{n}\right|^{2}\right)^{\beta-\frac{q(2+\alpha)}{p}} \int_{\Delta\left(z_{n}, \delta\right)}\left|(a-1) \overline{z_{n}} g(z)+g^{\prime}(z)\left(1-\overline{z_{n}} z\right)\right|^{q} d A(z) \leq C\left(\sum_{n=0}^{\infty}\left|b_{n}\right|^{p}\right)^{\frac{q}{p}} .
$$

Since $a+1>a>(2+\alpha) \max \left\{1, \frac{1}{p}\right\}$, Theorem 13 (ii) implies that setting

$$
h(z)=\sum_{j=0}^{\infty} \frac{b_{j}\left(1-\left|z_{j}\right|^{2}\right)^{a+1-\frac{2+\alpha}{p}}}{\left(1-\overline{z_{j}} z\right)^{a}}
$$

we have $\|h\|_{\mathcal{D}_{\alpha}^{p}} \approx\left\|\left\{b_{j}\right\}_{j=0}^{\infty}\right\|_{l^{p}}$. Arguing with $h$ in the same way as we have just argued with $f$, we obtain

$$
\sum_{n=0}^{\infty}\left|b_{n}\right|^{q}\left(1-\left|z_{n}\right|^{2}\right)^{\beta-\frac{q(2+\alpha)}{p}} \int_{\Delta\left(z_{n}, \delta\right)}\left|a \overline{z_{n}} g(z)+g^{\prime}(z)\left(1-\overline{z_{n}} z\right)\right|^{q} d A(z) \leq C\left(\sum_{n=0}^{\infty}\left|b_{n}\right|^{p}\right)^{\frac{q}{p}} .
$$

Using the last two inequalities, we easily deduce that

$$
\sum_{n=0}^{\infty}\left|b_{n}\right|^{q}\left(1-\left|z_{n}\right|^{2}\right)^{\beta-\frac{q(2+\alpha)}{p}}\left|z_{n}\right|^{q} \int_{\Delta\left(z_{n}, \delta\right)}|g(z)|^{q} d A(z) \leq C\left(\sum_{n=1}^{\infty}\left|b_{n}\right|^{p}\right)^{\frac{q}{p}} .
$$

Since the sequence $\left\{b_{n}\right\} \in \ell^{p}$ is arbitrary and $\frac{p}{q}>1$, using the duality $\left(l^{\frac{p}{q}}\right)^{\star}=l^{\frac{p}{p-q}}$ we obtain

$$
\sum_{n=0}^{\infty}\left(\left(1-\left|z_{n}\right|^{2}\right)^{\beta-\frac{q(2+\alpha)}{p}}\left|z_{n}\right|^{q} \int_{\Delta\left(z_{n}, \delta\right)}|g(z)|^{q} d A(z)\right)^{\frac{p}{p-q}}<\infty
$$


or, equivalently,

$$
\sum_{n=0}^{\infty}\left(\left|z_{n}\right|^{q}\left(1-\left|z_{n}\right|^{2}\right)^{\beta-2-\frac{q}{p} \alpha} \int_{\Delta\left(z_{n}, \delta\right)}|g(z)|^{q} d A(z)\right)^{\frac{p}{p-q}} A\left(\Delta\left(z_{n}, \delta\right)\right)<\infty .
$$

Now arguing as on pp. 337-338 of [27] we deduce that

$$
\int_{\mathbb{D}}\left(\left(1-|z|^{2}\right)^{\beta-2-\frac{q}{p} \alpha} \int_{\Delta(z, \delta)}|g(w)|^{q} d A(w)\right)^{\frac{p}{p-q}} d A(z)<\infty .
$$

By the subharmonicity of $|g|^{q}$ we have

$$
\left(1-|z|^{2}\right)^{2}|g(z)|^{q} \leq C \int_{\Delta(z, \delta)}|g(w)|^{q} d A(w) .
$$

Using this in (4.3) yields

$$
\int_{\mathbb{D}}|g(z)|^{\frac{q p}{p-q}}\left(1-|z|^{2}\right)^{\frac{p \beta-q \alpha}{p-q}} d A(z)<\infty
$$

that is,

$$
g \in A_{\frac{p \beta-q \alpha}{p-q}}^{\frac{q p}{p-q}} \text { if } \frac{p \beta-q \alpha}{p-q}>-1 \text { and } g \equiv 0 \text { if } \frac{p \beta-q \alpha}{p-q} \leq-1 .
$$

Thus, we have proved part (2) of Theorem 1 and the equivalence $(2) \Leftrightarrow(3)$ in Theorem 2 as desired.

Part (3) of Theorem 1 follows from Theorem 12. Indeed, it is clear from the definition that $J_{g}$ maps $\mathcal{D}_{\alpha}^{p}$ to $\mathcal{D}_{\beta}^{q}$ if and only if the measure $\mu_{g, q, \beta}$ defined by

$$
d \mu_{g, q, \beta}(z)=\left|g^{\prime}(z)\right|^{q}\left(1-|z|^{2}\right)^{\beta} d A(z)
$$

is a $\left(\mathcal{D}_{\alpha}^{p}, q\right)$-Carleson measure. Using Theorem 12 and (3.7) we see that this implies that we have, for a certain $\delta>0$,

$$
\int_{\mathbb{D}}\left(1-|z|^{2}\right)^{\frac{p \beta-q \alpha+p q}{p-q}}\left(\frac{1}{\left(1-|z|^{2}\right)^{2}} \int_{\Delta(z, \delta)}\left|g^{\prime}(\xi)\right|^{q} d A(\xi)\right)^{p /(p-q)} d A(z)<\infty .
$$

This and the subharmonicity of $\left|g^{\prime}\right|^{q}$ imply

$$
\int_{\mathbb{D}}\left(1-|z|^{2}\right)^{\frac{p \beta-q \alpha+p q}{p-q}}\left|g^{\prime}(z)\right|^{\frac{p q}{p-q}} d A(z)<\infty,
$$

that is, $g^{\prime} \in A_{\frac{p q+p \beta-q \alpha}{p-q}}^{\frac{q p}{p-q}}$. Since $\frac{p \beta-q \alpha}{p-q}>-1$, this implies that $g \in A_{\frac{p \beta-q \alpha}{p-q}}^{\frac{q p}{p-q}}$.

Finally, we note that part 4 of Theorem 1 follows readily from parts 1,2 and 3 and (1.1).

\section{A proof of Theorem 2 indePendent of the Decomposition theorem AND A PROOF OF THEOREM 5}

In this section we shall present a proof of Theorem 2 which makes no use of our decomposition theorem. Instead, we shall use a number of results concerning power series with Hadamard gaps, the sets of zeros of functions in the Dirichlet spaces and, also, arguments involving the use of Rademacher functions.

Let us list the results which will be used in our proof. The following result is due to Gnuschke [20, Theorems 1 and 2]. 
Theorem I. Suppose that $b>0, a>-1$ and $f$ is an analytic function in $\mathbb{D}$ which is given by a power series with Hadamard gaps, $f(z)=\sum_{k=1}^{\infty} a_{k} z^{n_{k}}(z \in \mathbb{D})$. Then

$$
\sum_{k=1}^{\infty}\left|a_{k}\right|^{b} n_{k}^{-(a+1)} \approx \int_{0}^{1}(1-r)^{a}\left|f\left(r e^{i t}\right)\right|^{b} d r, \quad \text { for all } t \in \mathbb{R} .
$$

We shall also use the following results of Girela and Peláez [16, Theorems 1.6 and 1.7] concerning the sets of zeros of functions in the spaces $\mathcal{D}_{p-1}^{p}$.

Theorem J. Suppose that $2<p<\infty$ and let $f$ be a function which belongs to the space $\mathcal{D}_{p-1}^{p}$ with $f(0) \neq 0$. Let $\left\{z_{k}\right\}_{k=1}^{\infty}$ be the sequence nonzero zeros of $f$ ordered so that $\left|z_{k}\right| \leq\left|z_{k+1}\right|$, for all $k$. Then

$$
\prod_{k=1}^{N} \frac{1}{\left|z_{k}\right|}=\mathrm{o}\left((\log N)^{\frac{1}{2}-\frac{1}{p}}\right), \quad \text { as } N \rightarrow \infty .
$$

Theorem K. If $2<p<\infty$ and $0<\gamma<\frac{1}{2}-\frac{1}{p}$, then there exists a function $f \in \mathcal{D}_{p-1}^{p}$ with $f(0) \neq 0$ such that if $\left\{z_{k}\right\}_{k=1}^{\infty}$ is the sequence of ordered nonzero zeros of $f$, then

$$
\prod_{k=1}^{N} \frac{1}{\left|z_{k}\right|} \neq \mathrm{o}\left((\log N)^{\gamma}\right), \quad \text { as } N \rightarrow \infty .
$$

Now we can proceed to present the announced proof. Let us remark that the proof we gave above of the equivalence $(1) \Leftrightarrow(3)$ in Theorem 2 did not use Theorem 13. Hence we only have to prove the implication $(2) \Rightarrow(3)$. For the sake of simplicity, we shall split this into several cases. The first three correspond to those where $q=\beta-1$ and $p=\alpha-1$ and will be grouped in the following theorem.

Theorem 14. Suppose that $0<q<p$ and let $g$ be an analytic function in $\mathbb{D}$. If $g \in M\left(\mathcal{D}_{p-1}^{p}, \mathcal{D}_{q-1}^{q}\right)$, then $g \equiv 0$.

Proof. We shall split the proof into several cases.

Case 1: $q>2$. Suppose that $g \in M\left(\mathcal{D}_{p-1}^{p}, \mathcal{D}_{q-1}^{q}\right)$ and $g \not \equiv 0$. Set $\gamma=\frac{1}{2}-\frac{1}{q}$. Notice that $0<\gamma<\frac{1}{2}-\frac{1}{p}$. Then let $f \not \equiv 0$ be the $\mathcal{D}_{p-1}^{p}$-function constructed in Theorem $\mathrm{K}$ for such a $\gamma$. Let $\left\{z_{k}\right\}_{k=1}^{\infty}$ be the sequence of ordered nonzero zeros of $f$ and let $\left\{w_{k}\right\}_{k=1}^{\infty}$ be the sequence of ordered nonzero zeros of $f g$. We have

$$
\prod_{k=1}^{N} \frac{1}{\left|z_{k}\right|} \neq \mathrm{o}\left((\log N)^{\gamma}\right), \quad \text { as } N \rightarrow \infty .
$$

On the other hand, since $f g \in \mathcal{D}_{q-1}^{q}$, Theorem \implies that

$$
\prod_{k=1}^{N} \frac{1}{\left|w_{k}\right|}=\mathrm{o}\left((\log N)^{\gamma}\right), \quad \text { as } N \rightarrow \infty .
$$

But this is a contradiction because any zero of $f$ is also a zero of $f g$. Thus

$$
g \in M\left(\mathcal{D}_{p-1}^{p}, \mathcal{D}_{q-1}^{q}\right) \Rightarrow g \equiv 0 .
$$

Case 2: $q \leq 2<p$. The proof is similar to that of the previous case. Suppose that $g \not \equiv 0$ and $g \in M\left(\mathcal{D}_{p-1}^{p}, \mathcal{D}_{q-1}^{q}\right)$. Set $\gamma=\frac{1}{2}-\frac{1}{q}<\frac{1}{2}-\frac{1}{p}$. Then let $f \not \equiv 0$ be the $\mathcal{D}_{p-1}^{p}$-function constructed in Theorem $\mathrm{K}$ for such a $\gamma$. Let $\left\{z_{k}\right\}_{k=1}^{\infty}$ be the sequence 
of ordered nonzero zeros of $f$ and let $\left\{w_{k}\right\}_{k=1}^{\infty}$ be the sequence of ordered nonzero zeros of $f g$. The sequence $\left\{z_{k}\right\}_{k=1}^{\infty}$ satisfies (5.3). Since $f g \in \mathcal{D}_{q-1}^{q}$ and $q \leq 2$, it follows that $f g \in H^{q}$ and, hence, $\left\{w_{k}\right\}_{k=1}^{\infty}$ satisfies the Blaschke condition, which is equivalent to saying that

$$
\prod_{k=1}^{N} \frac{1}{\left|w_{k}\right|}=\mathrm{O}(1), \quad \text { as } N \rightarrow \infty
$$

Again, this is in contradiction with (5.3) because any zero of $f$ is also a zero of $f g$. Consequently, $g$ must be identically zero.

Case 3: $q<p \leq 2$. Suppose that $g \in M\left(\mathcal{D}_{p-1}^{p}, \mathcal{D}_{q-1}^{q}\right)$ and $g \not \equiv 0$. Take $f(z)=$ $\sum_{k=0}^{\infty} a_{k} z^{2^{k}} \in \mathcal{D}_{p-1}^{p} \backslash \mathcal{D}_{q-1}^{q}$; that is, we have

$$
\sum_{k=0}^{\infty}\left|a_{k}\right|^{p}<\infty \text { and } \sum_{k=0}^{\infty}\left|a_{k}\right|^{q}=\infty .
$$

Let $\left\{r_{k}(t)\right\}_{k=0}^{\infty}$ be the sequence of Rademacher functions and denote

$$
f_{t}(z)=\sum_{k=0}^{\infty} r_{k}(t) a_{k} z^{2^{k}}, \quad 0 \leq t \leq 1, z \in \mathbb{D} .
$$

By Proposition $\mathrm{A}$,

$$
\left\|f_{t}\right\|_{H^{2}}^{2 p}=\left(\sum_{k=0}^{\infty}\left|a_{k}\right|^{2}\right)^{p} \leq\left(\sum_{k=0}^{\infty}\left|a_{k}\right|^{p}\right)^{2} \approx\left\|f_{t}\right\|_{\mathcal{D}_{p-1}^{p}}^{2 p} \approx\|f\|_{\mathcal{D}_{p-1}^{p}}^{2 p}<\infty,
$$

for all $t$, and then it follows that

$$
\int_{\mathbb{D}}\left|\left(g f_{t}\right)^{\prime}(z)\right|^{q}\left(1-|z|^{2}\right)^{q-1} d A(z) \leq C|| f_{t}\left\|_{\mathcal{D}_{p-1}^{p}}^{q} \approx\right\| f \|_{\mathcal{D}_{p-1}^{p}}^{q} .
$$

Using Fubini's Theorem, Khinchine's inequality, (5.4), (5.5) and the fact that $g \in$ $\mathcal{D}_{q-1}^{q}$, we obtain

$$
\begin{aligned}
& \int_{0}^{1} \int_{\mathbb{D}}\left|g f_{t}^{\prime}(z)\right|^{q}\left(1-|z|^{2}\right)^{q-1} d A(z) d t \\
& \leq C\left(\int_{0}^{1} \int_{\mathbb{D}}\left|\left(g f_{t}\right)^{\prime}(z)\right|^{q}\left(1-|z|^{2}\right)^{q-1} d A(z) d t+\int_{0}^{1} \int_{\mathbb{D}}\left|\left(g^{\prime} f_{t}\right)(z)\right|^{q}\left(1-|z|^{2}\right)^{q-1} d A(z) d t\right) \\
& \leq C\left(\|f\|_{\mathcal{D}_{p-1}^{p}}^{q}+\int_{\mathbb{D}}\left|g^{\prime}(z)\right|^{q}\left(\int_{0}^{1}\left|f_{t}(z)\right|^{q} d t\right)\left(1-|z|^{2}\right)^{q-1} d A(z) d t\right) \\
& \leq C\left(\|f\|_{\mathcal{D}_{p-1}^{p}}^{q}+\int_{\mathbb{D}}\left|g^{\prime}(z)\right|^{q} M_{2}(|z|, f)^{q}\left(1-|z|^{2}\right)^{q-1} d A(z) d t\right) \\
& \leq C\left(\|f\|_{\mathcal{D}_{p-1}^{p}}^{q}+\|f\|_{\mathcal{D}_{p-1}^{p}}^{q} \int_{\mathbb{D}}\left|g^{\prime}(z)\right|^{q}\left(1-|z|^{2}\right)^{q-1} d A(z) d t\right) \\
& \leq C\|f\|_{\mathcal{D}_{p-1}^{p}}^{q}<\infty .
\end{aligned}
$$

On the other hand, since $g \not \equiv 0$ there exists a positive constant $C$ such that

$$
M_{q}^{q}(r, g) \geq C, \quad 1 / 2<r<1 .
$$

Then, again using Fubini's Theorem and Khinchine's inequality and bearing in mind that $f^{\prime}$ is also given by a power series with Hadamard gaps (and, hence, 
$\left.M_{2}\left(r, f^{\prime}\right) \approx M_{q}\left(r, f^{\prime}\right)\right)$, we have that

$$
\begin{aligned}
& \int_{0}^{1} \int_{\mathbb{D}}\left|g f_{t}^{\prime}(z)\right|^{q}\left(1-|z|^{2}\right)^{q-1} d A(z) d t \\
& =\int_{\mathbb{D}}|g(z)|^{q}\left(1-|z|^{2}\right)^{q-1}\left(\int_{0}^{1}\left|f_{t}^{\prime}(z)\right|^{q} d t\right) \\
& \approx \int_{\mathbb{D}}|g(z)|^{q}\left(1-|z|^{2}\right)^{q-1} M_{2}\left(|z|, f^{\prime}\right)^{q} d A(z) \\
& \geq C \int_{1 / 2}^{1} M_{q}(r, g) M_{q}\left(r, f^{\prime}\right)^{q}\left(1-r^{2}\right)^{q-1} d r \\
& \geq C \int_{1 / 2}^{1} M_{q}\left(r, f^{\prime}\right)^{q}\left(1-r^{2}\right)^{q-1} d r=\infty .
\end{aligned}
$$

This is in contradiction with (5.6). Thus, it follows that $g \equiv 0$.

To finish our proof of Theorem 2 we still have to consider three cases. Recall that we are assuming that $0<q<p<\infty, \alpha, \beta>-1, p(\beta+1) \leq q(\alpha+1)$.

Case $A$ : $\beta<q-1$. Suppose that $g \in M\left(\mathcal{D}_{\alpha}^{p}, \mathcal{D}_{\beta}^{q}\right)$ and $g \not \equiv 0$. We have that $g \in D_{\beta}^{q}$. Let $f$ be an analytic function in $\mathbb{D}$ which is given by a power series with Hadamard gaps

$$
f(z)=\sum_{k=0}^{\infty} a_{k} z^{n_{k}}, \quad \frac{n_{k+1}}{n_{k}} \geq \lambda>1, \quad \text { for all } k
$$

with

$$
a_{k}=n_{k}^{-\frac{q-\beta-1}{q}} \text { if } p(\beta+1)<q(\alpha+1)
$$

and

$$
a_{k}=n_{k}^{-\frac{q-\beta-1}{q}} k^{-\gamma} \text { with } 1 / p<\gamma<1 / q \text { if } p(\beta+1)=q(\alpha+1) .
$$

Notice that we have that $\sum\left|a_{k}\right|<\infty$, which implies that $f \in H^{\infty}$.

Also, using Proposition $\mathrm{A}$ we deduce that $f \in D_{\alpha}^{p}$.

On the other hand, using the definition of the $a_{k}$ 's and Theorem 1 we see that

$$
\int_{0}^{1}(1-r)^{\beta}\left|f^{\prime}\left(r e^{i t}\right)\right|^{q} d r=\infty, \quad \text { for all } t .
$$

Since $f \in D_{\alpha}^{p}$ we have that $M_{g}(f) \in D_{\beta}^{q}$, that is,

$$
\int_{\mathbb{D}}(1-|z|)^{\beta}\left|f^{\prime}(z) g(z)+f(z) g^{\prime}(z)\right|^{q} d A(z)<\infty .
$$

Now, since $g \in D_{\beta}^{q}$ and $f \in H^{\infty}$, we have that

$$
\int_{\mathbb{D}}(1-|z|)^{\beta}\left|f(z) g^{\prime}(z)\right|^{q} d A(z)<\infty .
$$

Then it follows that

$$
\int_{\mathbb{D}}(1-|z|)^{\beta}\left|f^{\prime}(z) g(z)\right|^{q} d A(z)<\infty .
$$

Using the fact that $\beta<q-1$, it is a simple exercise to show that $\mathcal{D}_{\beta}^{q} \subset H^{q}$. Hence, $g \in H^{q}$, which implies that it has a finite and nonzero radial limit a.e. This and 
(5.7) imply that

$$
\int_{0}^{1}(1-r)^{\beta}\left|g\left(r e^{i t}\right) f^{\prime}\left(r e^{i t}\right)\right|^{q} d r=\infty, \quad \text { for almost every } t .
$$

This is in contradiction with (5.8). Hence, $g \equiv 0$.

Case B: $\beta=q-1$ and $p<\alpha+1$. Suppose $g \in M\left(\mathcal{D}_{\alpha}^{p}, \mathcal{D}_{\beta}^{q}\right)$ and $g \not \equiv 0$. Actually, we have $g \in M\left(A_{\alpha-p}^{p}, \mathcal{D}_{q-1}^{q}\right)$. Let $s$ be a positive number so large that $\alpha-p-\frac{2 p}{s}>-1$. Using the well-known fact that

$$
f \in A^{s} \Rightarrow M_{\infty}(r, f)=\mathrm{O}\left((1-r)^{-2 / s}\right)
$$

(see, e.g., [13, p. 80]) it follows readily that $A^{s} \subset A_{\alpha-p}^{p}$. Take $\eta \in\left(0, \frac{1}{s}\right)$. Now we use a well-known result of Horowitz [21] (see also Corollary 2 on p. 100 of [13]) to pick a function $f \in A^{s}$ (hence, $f \in A_{\alpha-p}^{p}$ ) with $f(0) \neq 0$ such that the sequence $\left\{z_{k}\right\}_{k=1}^{\infty}$ of ordered nonzero zeros of $f$ satisfies

$$
\prod_{k=1}^{N} \frac{1}{\left|z_{k}\right|} \geq C N^{\eta} .
$$

Since $g \in M\left(A_{\alpha-p}^{p}, \mathcal{D}_{q-1}^{q}\right)$ and $g \not \equiv 0$, we have that $g f \in \mathcal{D}_{q-1}^{q}$ and $g f \not \equiv 0$. Let $\left\{w_{k}\right\}_{k=1}^{\infty}$ be the sequence of ordered nonzero zeros of $f g$. Using the fact that $\mathcal{D}_{q-1}^{q} \subset H^{q}$ if $q \leq 2$ and Theorem $\rrbracket$ we see that

$$
\begin{array}{ll}
\prod_{k=1}^{N} \frac{1}{\left|w_{k}\right|}=\mathrm{o}\left((\log N)^{\frac{1}{2}-\frac{1}{p}}\right) \quad \text { if } q>2, \\
\prod_{k=1}^{N} \frac{1}{\left|w_{k}\right|}=\quad \mathrm{O}(1) \quad \text { if } q \leq 2 .
\end{array}
$$

Since any zero of $f$ is also a zero of $f g$, this is in contradiction with (5.9). Hence $g \equiv 0$.

Case $C: \beta>q-1$. In this case we also have $\alpha>p-1$, and then the problem reduces to showing that $M\left(A_{p-\alpha}^{p}, A_{q-\beta}^{q}\right)=0$. This was proved by Zhao [35, Theorem 1 (iv)].

Proof of Theorem 5. We split the proof into three cases.

Case 1: $p-1<\alpha$ and $\beta \leq p-1$. In this case $\mathcal{D}_{\alpha}^{p}=A_{\alpha-p}^{p}$ and then, using the above-mentioned result of Horowitz, we see that there exist $\eta>0$ and a function $f \in \mathcal{D}_{\alpha}^{p}$ such that $f(0) \neq 0$ such that the sequence $\left\{z_{k}\right\}_{k=1}^{\infty}$ of ordered nonzero zeros of $f$ satisfies

$$
\prod_{k=1}^{N} \frac{1}{\left|z_{k}\right|} \geq C N^{\eta}
$$


On the other hand, if $\left\{w_{k}\right\}_{k=1}^{\infty}$ is the sequence of ordered nonzero zeros of a function $h \in \mathcal{D}_{\beta}^{p}, h \not \equiv 0$, then:

- $\prod_{k=1}^{N} \frac{1}{\left|w_{k}\right|}=\mathrm{o}\left((\log N)^{\frac{1}{2}-\frac{1}{p}}\right)$ if $p>2$ and $\beta=p-1$, by Theorem Ð

and

- $\prod_{k=1}^{N} \frac{1}{\left|w_{k}\right|}=\mathrm{O}(1)$, if either $\beta<p-1$ or $\beta=p-1$ and $p \leq 2$, because in any of these two possibilities, $\mathcal{D}_{\beta}^{p} \subset H^{p}$.

Using these facts, and arguing as in cases 1 and 2 of Theorem 14, we deduce that $M\left(\mathcal{D}_{\alpha}^{p}, \mathcal{D}_{\beta}^{q}\right)=\{0\}$.

Case 2: $p>2$ and $\alpha=p-1>\beta$. This case can be handled as the previous one bearing in mind that $\mathcal{D}_{\beta}^{p} \subset H^{p}$ and that there are functions in $\mathcal{D}_{p-1}^{p}$ whose sequence of zeros does not satisfy the Blaschke condition (see Theorem $\mathrm{K}$ ).

Case 3: $p \leq 2$ and $\alpha=p-1>\beta$. Take $\left\{a_{k}\right\}_{k=0}^{\infty}$ to be a sequence of complex numbers with $\sum_{k=0}^{\infty}\left|a_{k}\right|^{p}<\infty$ and $\sum_{k=0}^{\infty} 2^{k(p-\beta-1)}\left|a_{k}\right|^{p}=\infty$ and set $f(z)=$ $\sum_{k=0}^{\infty} a_{k} z^{2^{k}}(z \in \mathbb{D})$. Using Theorem $₫$ we see that $f \in \mathcal{D}_{p-1}^{p} \backslash \mathcal{D}_{\beta}^{p}$. Let $\left\{r_{k}(t)\right\}_{k=0}^{\infty}$ be the sequence of Rademacher functions and denote

$$
f_{t}(z)=\sum_{k=0}^{\infty} r_{k}(t) a_{k} z^{2^{k}}, \quad 0 \leq t \leq 1, z \in \mathbb{D} .
$$

Arguing as in the proof of case 3 of Theorem 14 we obtain that if $g \in M\left(\mathcal{D}_{p-1}^{p}, \mathcal{D}_{\beta}^{p}\right)$, then

$$
\int_{0}^{1} \int_{\mathbb{D}}\left|g f_{t}^{\prime}(z)\right|^{p}\left(1-|z|^{2}\right)^{\beta} d A(z) d t \leq C\|f\|_{\mathcal{D}_{p-1}^{p}}^{p}<\infty .
$$

On the other hand, if $g$ were not identically zero, then there would exist a positive constant $C$ such that

$$
M_{p}^{p}(r, g) \geq C, \quad 1 / 2<r<1,
$$

and then, arguing again as in the proof of case 3 of Theorem 14, it would follow that

$$
\int_{0}^{1} \int_{\mathbb{D}}\left|g f_{t}^{\prime}(z)\right|^{p}\left(1-|z|^{2}\right)^{\beta} d A(z) d t=\infty .
$$

This is in contradiction with (5.10). Thus $g$ must be identically zero.

\section{Power series with Hadamard gaps as multipliers}

This section is devoted to proving Theorems 3, 4, 6 and 7 .

We shall use several results in our proof. The first one is a generalization of Theorem 3.2 of [18, and the second is an extension of Theorem 3.3 of [18. Both can be proved with the arguments used in 18 . We omit the details.

Theorem 15. Suppose that $0<p<\infty, \alpha>-1$ and let $g$ be an analytic function in $\mathbb{D}$ which is given by a power series with Hadamard gaps,

$$
g(z)=\sum_{k=1}^{\infty} a_{k} z^{n_{k}} \quad(z \in \mathbb{D}) \text { with } n_{k+1} \geq \lambda n_{k}, \text { for all } k \quad(\lambda>1) .
$$

Then the following conditions are equivalent:

(a) The measure $\mu_{g, p, \alpha}$ on $\mathbb{D}$ defined by $d \mu_{g, p \alpha}=\left(1-|z|^{2}\right)^{\alpha}\left|g^{\prime}(z)\right|^{p} d A(z)$ is a classical Carleson measure.

(b) $g \in \mathcal{D}_{\alpha}^{p}$. 
(c) $\sum_{n=0}^{\infty} 2^{-n(\alpha+1-p)}\left(\sum_{k=2^{n}}^{2^{n+1}-1}\left|a_{k}\right|\right)^{p}<\infty$.

(d) $\sum_{n=0}^{\infty} 2^{-n(\alpha+1-p)}\left(\sum_{k=2^{n}}^{2^{n+1}-1}\left|a_{k}\right|^{p}\right)<\infty$.

Lemma 1. Suppose that $0<q<\infty, \beta>-1$ and let $\phi$ be a positive and increasing function defined in $(0,1)$ such that

$$
\int_{0}^{1}(1-r)^{\beta} \phi^{q}(r) d r<\infty .
$$

Then there exists a function $g \in \mathcal{D}_{\beta}^{q}$ given by a power series with Hadamard gaps such that

$$
M_{2}\left(r, g^{\prime}\right) \geq \phi(r) \quad \text { for all } r \in(0,1) .
$$

Lemma 2 below can be proved with the arguments used in the proof of the first implication of Theorem 6 of [28]. Once more, we omit the details.

Lemma 2. Suppose that $0<A<\infty,-1<B<\infty, 0<p<\infty$ and $g(z)=$ $\sum_{k=0}^{\infty} a_{k} z^{k} \in \mathcal{H}$ ol $(\mathbb{D})$. Then

$$
\int_{0}^{1} M_{2}\left(r, g^{\prime}\right)^{p}(1-r)^{B}\left(\log \frac{e}{1-r}\right)^{A} d r \geq C \sum_{n=0}^{\infty} n^{A} 2^{-n(B+1-p)}\left(\sum_{k=2^{n}}^{2^{n+1}}\left|a_{k}\right|^{2}\right)^{p / 2} .
$$

Lemma 3. Suppose that $0<\beta<\infty$ and $0<p<\infty$. Then there exists a function $g \in H^{\infty}$ which is given by a power series with Hadamard gaps and satisfies

$$
\int_{0}^{1} M_{2}\left(r, g^{\prime}\right)^{p}(1-r)^{p-1}\left(\log \frac{e}{1-r}\right)^{\beta} d r=\infty .
$$

Proof. Take $\alpha \in(1, \infty)$ and set

$$
g(z)=\sum_{j=1}^{\infty}\left(j \log ^{\alpha} j\right)^{-1} z^{2^{2^{j}}}=\sum_{k=0}^{\infty} a_{z} z^{k}, \quad z \in \mathbb{D} .
$$

Since $\sum_{j=1}^{\infty}\left(j \log ^{\alpha} j\right)^{-1}<\infty, g \in H^{\infty}$. Moreover, using Lemma 2] we obtain

$$
\begin{aligned}
& \int_{0}^{1} M_{2}\left(r, g^{\prime}\right)^{p}(1-r)^{p-1}\left(\log \frac{e}{1-r}\right)^{\beta} d r \\
& \geq C \sum_{n=0}^{\infty} n^{\beta}\left(\sum_{k=2^{n}}^{2^{n+1}-1}\left|a_{k}\right|^{2}\right)^{p / 2} \\
& \geq C \sum_{j=0}^{\infty} \frac{2^{\beta j}}{j^{p} \log ^{p \alpha} j}=\infty .
\end{aligned}
$$

Now we turn to prove the announced results.

Proof of Theorem 3. Since $\mathcal{D}_{\beta}^{q}$ contains the constant functions, we certainly have that

$$
g \in M\left(\mathcal{D}_{\alpha}^{p}, \mathcal{D}_{\beta}^{q}\right) \Rightarrow g \in \mathcal{D}_{\beta}^{q} .
$$

Hence we have to prove the other implication.

Consider first the case $\alpha=p-1, p \leq 2$. Notice that then we have that $\beta>q-1$. Take $g \in \mathcal{D}_{\beta}^{p}$ given by a power series with Hadamard gaps. Using Theorem [15, we deduce that the measure $\mu$ defined by $d \mu(z)=d \mu_{g, q, \beta}(z)=\left(1-|z|^{2}\right)^{\beta}\left|g^{\prime}(z)\right|^{q} d A(z)$ 
is a classical Carleson measure and, hence, an $\left(H^{q}, q\right)$-Carleson measure. Since $0<q<p \leq 2$, we have that $\mathcal{D}_{p-1}^{p} \subset H^{p} \subset H^{q}$ and then it follows that $\mu$ is a $\left(\mathcal{D}_{p-1}^{p}, q\right)$-Carleson measure, which is equivalent to saying that $J_{g}\left(\mathcal{D}_{p-1}^{p}\right) \subset \mathcal{D}_{\beta}^{q}$. Then part 4 of Theorem 1 gives that $g \in M\left(\mathcal{D}_{p-1}^{p}, \mathcal{D}_{\beta}^{q}\right)$.

The case $p-2 \leq \alpha<p-1$ can be handled in a similar way using that $\mathcal{D}_{\alpha}^{p} \subset H^{p}$. However, we present a proof of this case based upon Arcozzi's characterization of $\left(\mathcal{D}_{\alpha}^{p}, q\right)$-Carleson measures in terms of Wolff potentials (Theorem $\mathbb{F}$ ). Hence, suppose that $p-2 \leq \alpha<p-1$ and take $g \in \mathcal{D}_{\beta}^{q}$ given by a power series with Hadamard gaps. As above, we have that the measure $\mu_{g, q, \beta}$ is a classical Carleson measure. Using this and the fact that $p-\alpha-1>0$, we deduce that, setting $\rho(z)=\left(1-|z|^{2}\right)^{\alpha-p+2}$, we have

$$
\begin{aligned}
W_{\rho, p}(\mu)(z) & =\int_{[0, z]}\left(\frac{\mu(S(w))}{\left(1-|w|^{2}\right)^{\alpha-p+2}}\right)^{p^{\prime}-1} \frac{|d w|}{1-|w|^{2}} \\
& \leq C \int_{[0, z]}\left(1-|w|^{2}\right)^{(p-\alpha-1)\left(p^{\prime}-1\right)} \frac{|d w|}{1-|w|^{2}} \\
& \leq C<\infty
\end{aligned}
$$

and this implies that

$$
\int_{\mathbb{D}}\left(W_{\rho, p}(\mu)(z)\right)^{\frac{q(p-1)}{p-q}} d \mu(z) \leq C \int_{\mathbb{D}}\left(1-|z|^{2}\right)^{\beta}\left|g^{\prime}(z)\right|^{q} d A(z)<\infty .
$$

Then, using Theorem $\mathrm{E}$, we deduce that $\mu$ is a $\left(\mathcal{D}_{\alpha}^{p}, q\right)$-Carleson measure. This implies that $g \in M\left(\mathcal{D}_{\alpha}^{p}, \mathcal{D}_{\beta}^{q}\right)$.

Proof of Theorem 4. Take $\varepsilon>0$ and $\eta>0$ such that $q\left(\frac{1}{2}-\frac{1}{p}-\varepsilon\right)>\eta$. Set

$$
f(z)=\sum_{k=0}^{\infty} \frac{1}{k^{\frac{1}{p}+\varepsilon}} z^{2^{k}}, \quad z \in \mathbb{D} .
$$

Using Proposition $\mathrm{A}$ we see that $f \in \mathcal{D}_{p-1}^{p}$. Also, it is easy to see that there exist $r_{0} \in(0,1)$ and $C>0$ such that

$$
M_{2}(r, f) \geq C\left(\log \frac{1}{1-r}\right)^{\frac{1}{2}-\frac{1}{p}-\varepsilon}, \quad r_{0} \leq r<1
$$

Also set

$$
\phi(r)=\frac{1}{(1-r)^{\frac{\beta+1}{q}}\left[\log \left(\frac{e}{1-r}\right)\right]^{\frac{1+\eta}{q}}}, \quad 0 \leq r<1 .
$$

Using Lemma 1, we see that there exists $g \in \mathcal{D}_{\beta}^{p}$ given by a power series with Hadamard gaps such that

$$
M_{2}\left(r, g^{\prime}\right) \geq \phi(r), \quad 0<r<1 .
$$

Take such a $g$. Arguing as in the proof of Theorem 2.1 of [18 we deduce that, setting $s=1+\eta-q\left(\frac{1}{2}-\frac{1}{p}-\varepsilon\right)$, we have

$$
\int_{r_{0}}^{1} \frac{d r}{(1-r) \log ^{s} \frac{e}{1-r}} d r \leq C \int_{\mathbb{D}}\left(1-|z|^{2}\right)^{\beta}\left|g^{\prime}(z)\right|^{q}|f(z)|^{q} d A(z) .
$$

Since $s<1$, it follows that $\int_{\mathbb{D}}\left(1-|z|^{2}\right)^{\beta}\left|g^{\prime}(z)\right|^{q}|f(z)|^{q} d A(z)=\infty$, that is, $J_{g}(f) \notin$ $\mathcal{D}_{\beta}^{q}$. Using Theorem 1 (iii), we deduce that $g \notin M\left(\mathcal{D}_{p-1}^{p}, \mathcal{D}_{\beta}^{q}\right)$. 
Proof of Theorem [6. (i) follows easily from the identity $M\left(A_{\alpha-p}^{p}\right)=H^{\infty}$.

Next we shall prove (ii). Hence, suppose that $0<p<\infty, \alpha<p-1$ and $g \in \mathcal{D}_{\alpha}^{p}$. Using Theorem [15 we see that $\mu_{g, p, \alpha}$ is a classical Carleson measure, that is, so $H^{p} \subset L^{p}\left(d \mu_{g, p, \alpha}\right)$. This and the fact that $\mathcal{D}_{\alpha}^{p} \subset H^{p}, \alpha+1<p$, gives that $\mathcal{D}_{\alpha}^{p} \subset L^{p}\left(d \mu_{g, p, \alpha}\right)$, that is, $\mu_{g, p, \alpha}$ is a $p$-Carleson measure for $\mathcal{D}_{\alpha}^{p}$. Next we shall prove that $g \in H^{\infty}$. By Theorem $\mathbb{C}$ (i), this finishes the proof.

Suppose first that $0<p \leq 1$. We have

$$
\begin{aligned}
\sum_{k=1}^{\infty}\left|a_{k}\right| & =\sum_{n=0}^{\infty}\left(\sum_{k=2^{n}}^{2^{n+1}-1}\left|a_{k}\right|\right) \\
& \leq C \sum_{n=0}^{\infty}\left(\sum_{k=2^{n}}^{2^{n+1}-1}\left|a_{k}\right|^{p}\right) \\
& \leq C \sum_{n=0}^{\infty} 2^{-n(\alpha+1-p)}\left(\sum_{k=2^{n}}^{2^{n+1}-1}\left|a_{k}\right|^{p}\right) .
\end{aligned}
$$

Then, using part (d) of Theorem [15, we deduce that $\sum_{k=1}^{\infty}\left|a_{k}\right|<\infty$ and, hence, $g \in H^{\infty}$.

Suppose now that $1<p<\infty$. Using part (c) of Theorem 15 and the Hölder inequality, we obtain

$$
\begin{aligned}
\sum_{k=1}^{\infty}\left|a_{k}\right| & =\sum_{n=0}^{\infty} 2^{-\frac{n(\alpha+1-p)}{p}} 2^{\frac{n(\alpha+1-p)}{p}}\left(\sum_{k=2^{n}}^{2^{n+1}-1}\left|a_{k}\right|\right) \\
& \leq C\left(\sum_{n=0}^{\infty} 2^{-n(\alpha+1-p)}\left(\sum_{k=2^{n}}^{2^{n+1}-1}\left|a_{k}\right|\right)^{p}\right)^{1 / p}\left(\sum_{n=0}^{\infty} 2^{\frac{n(\alpha+1-p)}{p-1}}\right)^{1-1 / p} \\
& \leq C\left(\sum_{n=0}^{\infty} 2^{-n(\alpha+1-p)}\left(\sum_{k=2^{n}}^{2^{n+1}-1}\left|a_{k}\right|\right)^{p}\right)^{1 / p}<\infty,
\end{aligned}
$$

which implies that $g \in H^{\infty}$.

(iii) and (iv) can be proved using Theorem 3.2 and Theorem D of [18 and arguments similar to those used in the proof of (ii). We omit the details.

Proof of Theorem 7. Suppose that $2<p<\infty$. Take a positive number $\varepsilon$ such that $0<\varepsilon<\frac{1}{2}-\frac{1}{p}$ and define

$$
f(z)=\sum_{k=0}^{\infty} \frac{1}{k^{\frac{1}{p}+\varepsilon}} z^{2^{k}}, \quad z \in \mathbb{D} .
$$

Using Proposition $₫$, we see that $f \in \mathcal{D}_{p-1}^{p}$. Also, it is easy to see that there exist $r_{0} \in(0,1)$ and $C>0$ such that

$$
M_{2}(r, f) \geq C\left(\log \frac{1}{1-r}\right)^{\frac{1}{2}-\frac{1}{p}-\varepsilon}, \quad r_{0} \leq r<1 .
$$


Arguing as in the proof of Theorem 2.1 of $[18$, we see that for any $g \in \mathcal{H o l}(\mathbb{D})$ given by a power series with Hadamard gaps, we have

$$
\begin{gathered}
\int_{\mathbb{D}}\left(1-|z|^{2}\right)^{p-1}\left|g^{\prime}(z)\right|^{p}|f(z)|^{p} d A(z) \\
\geq C \int_{r_{0}}^{1}(1-r)^{p-1} M_{2}^{p}\left(r, g^{\prime}\right)\left(\log \frac{1}{1-r}\right)^{\frac{p}{2}-1-p \varepsilon} d r .
\end{gathered}
$$

Then, if we let $g$ be the function constructed in Lemma 3 with $\beta=\frac{p}{2}-1-p \varepsilon$, we have that

$$
\int_{\mathbb{D}}\left(1-|z|^{2}\right)^{p-1}\left|g^{\prime}(z)\right|^{p}|f(z)|^{p} d A(z)=\infty,
$$

which together with part (i) of Theorem $\mathbb{C}$ gives that $g \notin M\left(\mathcal{D}_{p-1}^{p}\right)$. This finishes the proof.

\section{Compact multipliers of $\mathcal{D}_{p-1}^{p}$}

This section is devoted to proving Theorem 8 . We shall use the following lemma.

Lemma 4. For every $p \in(0, \infty)$ there exist two positive constants $C_{1}(p)$ and $C_{2}(p)$ such that

$$
C_{1} \leq\left\|z^{n}\right\|_{\mathcal{D}_{p-1}^{p}} \leq C_{2}, \quad \text { for all } n \in \mathbb{N} .
$$

Proof. Let $\Gamma($.$) and B(.,$.$) be the classical gamma and beta functions. Using Stir-$ ling's formula we obtain

$$
\begin{aligned}
\left\|z^{n}\right\|_{\mathcal{D}_{p-1}^{p}}^{p} & =C n^{p} \int_{0}^{1} r^{(n-1) p+1}(1-r)^{p-1} d r \\
& =C n^{p} B((n-1) p+2, p) \\
& =C n^{p} \frac{\Gamma((n-1) p+2) \Gamma(p)}{\Gamma(n p+2)} \\
& \asymp C n^{p} \frac{((n-1) p+2)^{(n-1) p+\frac{3}{2}}}{(n p+2)^{n p+\frac{3}{2}}} \\
& \asymp C .
\end{aligned}
$$

Proof of Theorem 8 . Suppose that $g \in \mathcal{H}$ ol $(\mathbb{D}), g \not \equiv 0$, and that $M_{g}$ is a compact operator from $\mathcal{D}_{p-1}^{p}$ to itself. Say that $g$ has a zero of order $N \geq 0$ at 0 and let $h$ be defined by $g(z)=z^{N} h(z)(z \in \mathbb{D})$. By Lemma 4 ,

$$
\sup _{n \in \mathbb{N}}\left\|g z^{n}\right\|_{\mathcal{D}_{p-1}^{p}}=\sup _{n \in \mathbb{N}}\left\|h z^{n+N}\right\|_{\mathcal{D}_{p-1}^{p}}<\infty,
$$

which, since $M_{g}$ is compact and the sequence $\left\{z^{n}\right\}_{n \in \mathbb{N}}$ converges uniformly to zero on compact subsets of $\mathbb{D}$, gives that there exists an increasing sequence of natural numbers $\left\{n_{k}\right\}$ such that

$$
\lim _{k \rightarrow \infty}\left\|h z^{N+n_{k}}\right\|_{\mathcal{D}_{p-1}^{p}}=0 .
$$


Notice that $h \in \mathcal{D}_{p-1}^{p}$ and then, by the dominated convergence theorem, we have that

$$
\begin{gathered}
\lim _{k \rightarrow \infty} \int_{\mathbb{D}}|z|^{p\left(N+n_{k}\right)}\left|h^{\prime}(z)\right|^{p}\left(1-|z|^{2}\right)^{p-1} d A(z) \\
=\int_{\mathbb{D}} \lim _{k \rightarrow \infty}\left(|z|^{p\left(N+n_{k}\right)}\left|h^{\prime}(z)\right|^{p}\left(1-|z|^{2}\right)^{p-1}\right) d A(z)=0 .
\end{gathered}
$$

Joining (7.2) and (7.3), we deduce that

$$
\lim _{k \rightarrow \infty} \int_{\mathbb{D}}\left|\left(N+n_{k}\right) z^{N+n_{k}-1} h(z)\right|^{p}(1-|z|)^{p-1} d A(z)=0
$$

or, equivalently,

$$
\lim _{k \rightarrow \infty}\left(N+n_{k}\right)^{p} \int_{0}^{1} r^{p\left(N+n_{k}-1\right)+1}(1-r)^{p-1} M_{p}^{p}(r, h) d r=0 .
$$

Since $h(0) \neq 0$, there exists a positive constant $C$ such that

$$
M_{p}^{p}(r, h) \geq C, \quad 0<r<1
$$

and then (7.4) implies

$$
\lim _{k \rightarrow \infty}\left(N+n_{k}\right)^{p} \int_{0}^{1} r^{p\left(N+n_{k}-1\right)+1}(1-r)^{p-1} d r=\lim _{k \rightarrow \infty}\left\|z^{N+n_{k}}\right\|_{\mathcal{D}_{p-1}^{p}}=0 .
$$

This is in contradiction with Lemma 4. Thus $g$ must be identically 0 .

\section{REFERENCES}

[1] A. Aleman, A class of integral operators on spaces of analytic functions, in: "Topics in Complex Analysis and Operator Theory" (D. Girela and C. González, eds.), University of Málaga, Spain, 2007, pp. 3-30. MR2394654

[2] A. Aleman and J. A. Cima, An integral operator on $H^{p}$ and Hardy's inequality, J. Anal. Math. 85 (2001), 157-176. MR.1869606 (2002k:30068)

[3] A. Aleman and A. G. Siskakis, Integration operators on Bergman spaces, Indiana Univ. Math. J. 46 (1997), no. 2, 337-356. MR.1481594 (99b:47039)

[4] J. Arazy, S. D. Fisher and J. Peetre, Möbius invariant function spaces, J. Reine Angew. Math. 363 (1985), 110-145. MR814017 (87f:30104)

[5] N. Arcozzi, R. Rochberg and E. Sawyer, Carleson measures for analytic Besov spaces, Rev. Mat. Iberoamericana 18 (2002), 443-510. MR1949836 (2003j:30080)

[6] N. Arcozzi, Carleson measures for analytic Besov spaces: the upper triangle case, J. Inequal. Pure and Appl. Math. 6 (2005), no. 1, 1-15. MR2122940 (2005k:30104)

[7] S. M. Buckley, P. Koskela and D. Vukotić, Fractional integration, differentiation, and weighted Bergman spaces, Math. Proc. Cambridge Philos. Soc. 126 (1999), 369-385. MR.1670257 (2000m:46050)

[8] L. Carleson, An interpolation problem for bounded analytic functions, Amer. J. Math. 80 (1958), 921-930. MR0117349 (22:8129)

[9] R. Coifman and R. Rochberg, Representation theorems for holomorphic and harmonic functions in $L^{p}$, Astérisque 77 (1980), 11-66. MR604369 (82j:32015)

[10] J. J. Donaire, D. Girela and D. Vukotić, On univalent functions in some Möbius invariant spaces, J. Reine Angew. Math. 553 (2002), 43-72. MR1944807 (2003k:30076)

[11] P. L. Duren, Extension of a Theorem of Carleson, Bull. Amer. Math. Soc. 75 (1969), 143-146. MR0241650 (39:2989)

[12] P. L. Duren, Theory of $H^{p}$ Spaces, Academic Press, New York-London, 1970. Reprint: Dover, Mineola, New York, 2000. MR0268655 (42:3552) 
[13] P. L. Duren and A. P. Schuster, Bergman Spaces, Math. Surveys and Monographs, Vol. 100, American Mathematical Society, Providence, Rhode Island, 2004. MR2033762 (2005c:30053)

[14] P. L. Duren, A. P. Schuster and D. Vukotić, On uniformly discrete sequences in the disk, in: "Quadrature domains and their applications", The Harold S. Shapiro Anniversary Volume (P. Ebenfelt, B. Gustafsson, D. Khavinson, and M. Putinar, editors) 131-150, Oper. Theory Adv. Appl., 156, Birkhäuser, Basel, 2005. MR2129739 (2005k:30105)

[15] T. M. Flett, The dual of an inequality of Hardy and Littlewood and some related inequalities, J. Math. Anal. Appl. 38 (1972), 756-765. MR0304667 (46:3799)

[16] D. Girela and J. A. Peláez, Growth properties and sequences of zeros of analytic functions in spaces of Dirichlet type, J. Australian Math. Soc. 80 (2006), 397-418. MR 2236047 (2007e:30037)

[17] D. Girela, M. Pavlović and J. A. Peláez, Spaces of analytic functions of Hardy-Bloch type, J. Anal. Math. 100 (2006), 53-81. MR2303304(2008a:30048)

[18] D. Girela and J. A. Peláez, Carleson measures for spaces of Dirichlet type, Integral Equations and Operator Theory 55 (2006), no. 3, 415-427. MR2244197(2007e:30079)

[19] D. Girela and J. A. Peláez, Carleson measures, multipliers and integration operators for spaces of Dirichlet type, J. Funct. Analysis 241 (2006), no. 1, 334-358. MR2264253 (2007m:46034)

[20] D. Gnuschke, Relations between certain sums and integrals concerning power series with Hadamard gaps, Complex Variables Theory Appl. 4 (1984), 89-100. MR770989 (86e:30004)

[21] C. Horowitz, Zeros of functions in the Bergman spaces, Duke Math. J. 41 (1974), 693-710. MR0357747 (50:10215)

[22] H. Hedenmalm, B. Korenblum and K. Zhu, Theory of Bergman Spaces, Graduate Texts in Mathematics, Vol. 199, Springer, New York, Berlin, 2000. MR.1758653 (2001c:46043)

[23] J. E. Littlewood and R. E. A. C. Paley, Theorems on Fourier series and power series. II, Proc. London Math. Soc. 42 (1936), 52-89.

[24] D. H. Luecking, Forward and reverse inequalities for functions in Bergman spaces and their derivatives, Amer. J. Math. 107 (1985), 85-111. MR778090 (86g:30002)

[25] D. H. Luecking, Multipliers of Bergman spaces into Lebesgue spaces, Proc. Edinburgh Math. Soc. (2) 29 (1986), 125-131. MR829188 (87e:46034)

[26] D. H. Luecking, Embedding derivatives of Hardy spaces into Lebesgue spaces, Proc. London Math. Soc. 63 (1991), no. 3, 595-619. MR.1127151 (92k:42030)

[27] D. H. Luecking, Embedding theorems for spaces of analytic functions via Khinchine's inequality, Michigan Math. J. 40 (1993), no. 2, 333-358. MR.1226835 (94e:46046)

[28] M. Mateljevic and M. Pavlovic, $L^{p}$ - behaviour of power series with positive coefficients and Hardy spaces, Proc. Amer. Math. Soc., 87 (1983), 309-316. MR681840 (84g:30034)

[29] M. M. Peloso, Möbius invariant spaces on the unit ball, Michigan Math. J. 39 (1992), 509536. MR.1182505 (93k:46018)

[30] R. Rochberg, Decomposition theorems for Bergman spaces and their applications, in: "Operator and function theory" (S. C. Power, ed.), Reidel, Dordrecht, The Netherlands, 1985, pp. 225-278. MR810448 (87c:46032)

[31] S. A. Vinogradov, Multiplication and division in the space of analytic functions with area integrable derivative, and in some related spaces (in Russian), Zap. Nauchn. Sem. S.-Peterburg. Otdel. Mat. Inst. Steklov. (POMI) 222 (1995), Issled. po Linein. Oper. i Teor. Funktsii 23, 45-77, 308; translation in J. Math. Sci. (New York) 87, no. 5 (1997), 3806-3827. MR 1359994 (96m:30053)

[32] R. Wallstén, Hankel operators between weighted Bergman spaces in the ball, Ark. Mat. 28 (1990), 182-192. MR1049650(91i:47041)

[33] Z. Wu and L. Yang, Multipliers between Dirichlet spaces, Integral Equations and Operator Theory 32 (1998), 482-492. MR1656473 (99j:47048)

[34] Z. Wu, Carleson measures and multipliers for Dirichlet spaces, J. Funct. Anal. 169 (1999), 148-163. MR1726750 (2000k:30081)

[35] R. Zhao, Pointwise multipliers from weighted Bergman spaces and Hardy spaces to weighted Bergman spaces, Ann. Acad. Sci. Fenn. 29 (2004), 139-150. MR2041703 (2004m:30059)

[36] K. Zhu, Operator Theory in Function Spaces, Marcel Dekker, New York, 1990. Reprint: Math. Surveys and Monographs, Vol. 138, American Mathematical Society, Providence, Rhode Island, 2007. MR.1074007(92c:47031) 
[37] K. Zhu, Analytic Besov spaces, J. Math. Anal. Appl. 157 (1991), 318-336. MR.1112319 (93e:30092)

[38] A. Zygmund, Trigonometric Series, Vol. I and Vol. II, Second edition, Camb. Univ. Press, Cambridge, 1959. MR0107776 (21:6498)

Department of Mathematics and Statistics, University of Cyprus, P.O. Box 20537, 1678 Nicosia, Cyprus

E-mail address: galanopoulos_petros@yahoo.gr

Current address: Departamento de Análisis Matemático, Universidad de Málaga, Facultad de Ciencias, Campus de Teatinos, 29071 Málaga, Spain

Departamento de Análisis Matemático, Universidad de Málaga, Campus de Teatinos, 29071 MÁlaga, Spain

E-mail address: girela@uma.es

Departamento de Matemáticas, Universidad de Córdoba, Edificio Einstein, Campus de Rabanales, 14014 Córdoba, Spain

E-mail address: ma1pemaj@uco.es

Current address: Departamento de Análisis Matemático, Universidad de Málaga, Facultad de Ciencias, Campus de Teatinos, 29071 Málaga, Spain

E-mail address: japelaez@uma.es 